International Journal of Pure and Applied Mathematics

Volume 96 No. 2 2014, 249-278

ISSN: 1311-8080 (printed version); ISSN: 1314-3395 (on-line version)

url: http://www.ijpam.eu

doi: http://dx.doi.org/10.12732/ijpam.v96i2.8

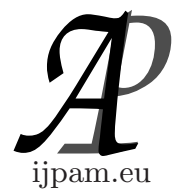

\title{
POSTULATION OF GENERAL UNIONS OF DOUBLE LINES, LINES AND DOUBLE POINTS IN A PROJECTIVE SPACE
}

\author{
E. Ballico \\ Department of Mathematics \\ University of Trento \\ 38123 Povo (Trento) - Via Sommarive, 14, ITALY
}

\begin{abstract}
We study the Hilbert function of general unions $X \subset \mathbb{P}^{r}$ of double points and a "small" number of lines and double lines. Non asymptotic results are given only for $r=3$.
\end{abstract}

AMS Subject Classification: 14N05, 14H99

Key Words: postulation, Hilbert function, fat point, unions of lines, double line

\section{Introduction}

For each $P \in \mathbb{P}^{r}, r \geq 3$, let $2 P$ be the closed subscheme of $\mathbb{P}^{r}$ with $\left(\mathcal{I}_{P}\right)^{2}$ as its ideal sheaf. We will say that $2 P$ is a 2 -point and that $P$ is the support of $2 P$. For any line $L \subset \mathbb{P}^{r}, r \geq 3$, let $2 L$ be the closed subscheme of $\mathbb{P}^{r}$ with $\left(\mathcal{I}_{L}\right)^{2}$ as its ideal sheaf. We will say that $2 L$ is a 2 -line and that $P$ is the support of $2 L$. Since the conormal bundle of $L$ is isomorphic to $\mathcal{O}_{L}(-1)^{\oplus(r-1)}, 2 L$ a scheme with $\chi\left(\mathcal{O}_{2 L}(t)\right)=r t+1$ for all $t \in \mathbb{Z}$.

For all $(t, a, c) \in \mathbb{N}^{3}$ and all $r \geq 3$ let $L(t, a, c ; r)$ denote the set of all schemes $X \subset \mathbb{P}^{r}$ which are the disjoint union of $t$ lines, a 2-points and $c$ 2-lines.

Received: June 14, 2014

(c) 2014 Academic Publications, Ltd. url: www.acadpubl.eu 
Theorem 1. Fix an integer $k \geq 5$ and $(t, a, c) \in \mathbb{N}^{3}$ such that $3 t+c \leq$ $\left\lfloor k^{2} / 64\right\rfloor$. Fix a general $X \in L(t, a, c ; 3)$.

(i) If $t(k+1)+(3 k+1) c+4 a \leq\left(\begin{array}{c}k+3 \\ 3\end{array}\right)$, then $h^{1}\left(\mathcal{I}_{X}(k)\right)=0$.

(ii) If $t(k+1)+(3 k+1) c+4 a \geq\left(\begin{array}{c}k+3 \\ 3\end{array}\right)$, then $h^{0}\left(\mathcal{I}_{X}(k)\right)=0$

Remark 1. Assume $3 t+c \leq\left\lfloor(k-1)^{2} / 64\right\rfloor$ and $t(k+1)+(3 k+1) c+4 a \leq$ $\left(\begin{array}{c}k+3 \\ 3\end{array}\right)$. Fix a general $X \in L(t, a, c ; 3)$. By part (i) for the integer $k$ and part (ii) for the integer $k-1$ we get that $X$ has maximal rank. The best constant we could obtain when $k \gg 0$ with the proof in this paper is $3 t+c \leq k^{2} / 32$, but we have numerical problems for low $k$. For a fixed $c \leq k^{2} / 32$ it is easy to get some $t$ with $3 t+c>k^{2} / 32$ (but not by much) and for which the proof works; in step (ii) of the proof of Theorem 1 we need to modify step (i2) adding a step $k-4 \Longrightarrow k-2$ in which we add lines in $Q$.

In section 3 we describe all cases with $k \leq 5$, the cases with $k=6$ and $c=1$ and a few other cases. There are a few cases with $h^{0}\left(\mathcal{I}_{X}(k)\right) \cdot h^{1}\left(\mathcal{I}_{X}(k)\right)>0$ for a general $X \in L(t, a, c ; 3)$, but each case may be explained by a geometrical reason for its bad Hilbert function in degree $k$.

In the case $r>4$ we first take the case $c=0$ with an additional (unknown) zero-dimensional scheme $Z$ (see [4], [6], [7] for the case $Z=\emptyset$ and/or $a t=0$ ).

Proposition 1. For all integers $r \geq 4$ and $z \geq 0$ there is an integer $\beta_{r, z}>0$ such that for every zero-dimensional scheme $Z \subset \mathbb{P}^{r}$ with $\operatorname{deg}(Z) \leq z$ and all $(t, a) \in \mathbb{N}^{2}$ with $a \geq \alpha_{r, x}$ a general union of $Z$ and a general element of $L(t, a, 0 ; r)$ has maximal rank.

Proposition 2. For all integers $r \geq 4$ and $z \geq 0$ there is an integer $\tau_{r, z}>0$ with the following property: for every zero-dimensional scheme $Z \subset \mathbb{P}^{r}$ with $\operatorname{deg}(Z) \leq z$ and all $(t, a) \in \mathbb{N}^{2}$ with $t \geq \tau_{r, z}$ a general union of $Z$ and a general element of $L(t, a, 0 ; r)$ has maximal rank.

Taking the union of Propositions 1 and 2 we get the following result.

Corollary 1. For all integers $r \geq 4$ and $z \geq 0$ there is an integer $\delta_{r, z}>0$ such that for every zero-dimensional scheme $Z \subset \mathbb{P}^{r}$ with $\operatorname{deg}(Z) \leq z$ and all $(t, a) \in \mathbb{N}^{2}$ with $a+t \geq \delta_{r, z}$ a general union of $Z$ and a general element of $L(t, a, 0 ; r)$ has maximal rank.

Remark 2. Fix an integer $x>0$ and assume $r \geq x+4$. Fix a degree $x$ integer-valued polynomial $\psi$ with $\operatorname{deg}(\psi)=x$ which is the Hilbert polynomial of some subscheme of $\mathbb{P}^{r}$. As in [5] we may use induction on $r$ and $x$ and get integers $\beta_{r, \psi}, \tau_{r, \psi}$ and $\delta_{r, \psi}$ which works as in Propositions 1, 2 and in Corollary 1. 
In the case $z=0$ and $c>0$ see Proposition 3.

Conjecture 1. Fix an integer $r \geq 3$. Is there an integer $u_{r}$ with the following property: fix $(t, a, c) \in \mathbb{N}^{3}$ such that $t+a \geq u_{r}$; has a general element of $L(t, a, c ; r)$ maximal rank?

We recall that [10] raised the question of the Hilbert function of unions of multiples of linear subspaces.

\section{Preliminaries}

For any finite subset $S \subset \mathbb{P}^{r}$ set $2 S:=\cup_{O \in S} 2 O$. For any finite union $E \subset \mathbb{P}^{r}$ of disjoint lines set $2 E:=\cup_{L \subseteq E} 2 L$. Fix a hyperplane $H \subset \mathbb{P}^{r}$. For any $P \in H$, any finite subset $S \subset H$, any line $L \subset H$ and any union $E \subset H$ of finitely may disjoint lines set $\{2 P, H\}::=2 P \cap H,\{2 S, H\}:=2 S \cap H,\{2 L, H\}:=2 L \cap H$ and $\{2 E, H\}:=2 E \cap H$. Write $L(t, a, c):=L(t, a, c ; 3)$ and call $X(t, a, c)$ a general element of $L(t, a, c)$.

For any closed subscheme $X \subset \mathbb{P}^{r}$ the residual scheme $\operatorname{Res}_{H}(X)$ of $X$ with respect to $H$ is the closed subscheme of $\mathbb{P}^{r}$ with $\mathcal{I}_{X}: \mathcal{I}_{H}$ as its ideal sheaf.

Let $X \subset \mathbb{P}^{r}$ be a closed subscheme. Fix a hypersurface $T \subset \mathbb{P}^{r}$ and set $y:=\operatorname{deg}(T)$. The residual scheme $\operatorname{Res}_{T}(X)$ of $X$ with respect to $t$ is the closed subscheme of $\mathbb{P}^{r}$ with $\mathcal{I}_{X}: \mathcal{I}_{T}$ as its ideal sheaf. For each $x \in \mathbb{Z}$ we have an exact sequence

$$
0 \rightarrow \mathcal{I}_{\operatorname{Res}_{T}(X)}(x-y) \rightarrow \mathcal{I}_{X}(x) \rightarrow \mathcal{I}_{X \cap T, T}(x) \rightarrow 0
$$

which is often called the Castelnuovo's sequence or the Horace sequence.

Let $Q \subset \mathbb{P}^{3}$ be a smooth quadric surface. For any $P \in Q$, any finite subset $S \subset H$, any line $L \subset H$ and any union $E \subset H$ of finitely may disjoint lines set $\{2 P, Q\}:=2 P \cap Q,\{2 S, Q\}:=2 S \cap Q,\{2 L, Q\}:=2 L \cap Q$ and $\{2 E, Q\}:=2 E \cap Q$. Call $\left|\mathcal{O}_{Q}(1,0)\right|$ and $\left|\mathcal{O}_{Q}(0,1)\right|$ the two rulings of $Q$. Fix $D \in\left|\mathcal{O}_{Q}(e, f)\right|$ and a closed subscheme $A$ of $Q$. The residual scheme $\operatorname{Res}_{D}(A)$ of $A$ with respect to $D$ is the closed subscheme of $Q$ with $\mathcal{I}_{A}: \mathcal{I}_{D}$ as its ideal sheaf. For all $(a, b) \in \mathbb{Z}^{2}$ we have an exact sequence (called again a Castelnuovo's sequence):

$$
0 \rightarrow \mathcal{I}_{\operatorname{Res}_{D}(A)}(a-e, b-f) \rightarrow \mathcal{I}_{A}(a, b) \rightarrow \mathcal{I}_{A \cap D, D}(a, b) \rightarrow 0
$$

Set $L(t, a, c):=L(t, a, c ; 3)$. For any $(t, a, b, c) \in \mathbb{N}^{3} \backslash\{(0,0,0)\}$ let $X(t, a, c)$ denote the general member of $L(t, a, c)$. We write $L(0,0,0):=\{\emptyset\}$. Therefore $X(0,0,0)=\emptyset$. For any $(t, a, c) \in \mathbb{N}^{3} \backslash\{(0,0,0)\}$ the minimal integer $k$ such that $(k+1) t+(3 k+1) c+4 a \leq\left(\begin{array}{c}k+3 \\ 3\end{array}\right)$ is called the critical value of $k$. We 
have $k=1$ if and only if $(t, a, b)$ is one of the following triples $(1,0,0),(2,0,0)$, $(0,1,0),(0,0,1)$. We claim that for any triple $(t, a, c)$ with critical value 1 the scheme $X(t, a, c)$ has maximal rank. To check the Hilbert function in the case $(t, a, c ; r)=(0,0,1 ; 3)$ it is sufficient to use the following well-known lemma.

Lemma 1. We have $h^{1}\left(\mathcal{I}_{X(0,0,1)}(x)\right)=0$ and $h^{0}\left(\mathcal{I}_{X(0,0,1)}(x)\right)=\left(\begin{array}{c}x+3 \\ 3\end{array}\right)-$ $3 x-1$ for all $t>0$.

We also use the following result ([4, Theorem 1]):

Lemma 2. $F$ ix $(t, a) \in \mathbb{N}^{2} \backslash\{(0,0)\}$ and an integer $k \geq 3$. Then either $h^{0}\left(\mathcal{I}_{X(t, a, 0)}(k)\right)=0$ or $h^{1}\left(\mathcal{I}_{X(t, a, 0)}(k)\right)=0$, unless either $(t, a, k)=(2,3,3)$ or $(t, a, k)=(0,9,4)$. We have $h^{0}\left(\mathcal{I}_{X(2,3,0)}(3)\right)=h^{0}\left(\mathcal{I}_{X(0,9,0)}(4)\right)=1$.

We use the following well-known and elementary lemma.

Lemma 3. Fix an integral surface $T \subset \mathbb{P}^{3}$, a closed subscheme $X \subset \mathbb{P}^{3}$ not containing $T$, integers $t>0, f>0$ and a general set $S \subset T$ such that $\sharp(S)=f$. Set $e:=\operatorname{deg}(T)$ and $u:=h^{0}\left(T, \mathcal{I}_{X \cap T}(t)\right)$.

(i) If $f \geq u$, then $h^{0}\left(\mathcal{I}_{X \cup S}(t)\right)=h^{0}\left(\mathcal{I}_{\operatorname{Res}_{T}(X)}(t-e)\right)$.

(ii) If $f \leq u, h^{1}\left(\mathcal{I}_{X}(t)\right)=0$ and $h^{0}\left(\mathcal{I}_{\operatorname{Res}_{T}(X)}(t-e)\right) \leq \max \left\{0, h^{0}\left(\mathcal{I}_{X}(t)\right)-\right.$ $f\}$, then $h^{1}\left(\mathcal{I}_{X \cup S}(t)\right)=0$.

(iii) If $h^{1}\left(\mathcal{I}_{X}(t)\right)=0$ and $h^{0}\left(\mathcal{I}_{\operatorname{Res}_{T}(X)}(t-e)\right) \leq \max \left\{0, h^{0}\left(\mathcal{I}_{X}(t)\right)-f\right\}$, then either $h^{0}\left(\mathcal{I}_{X \cup S}(t)\right)=0$ or $h^{1}\left(\mathcal{I}_{X \cup S}(t)\right)=0$.

Remark 3. Fix closed subschemes $Y \subset X \subset \mathbb{P}^{r}$ and $t \in \mathbb{N}$. If $h^{0}\left(\mathcal{I}_{Y}(t)\right)=$ 0 , then obviously $h^{0}\left(\mathcal{I}_{X}(t)\right)=0$. Now assume that $Y$ is the union of some of the connected components of $Y$. In this case the restriction map $H^{0}\left(\mathcal{O}_{X}(t)\right) \rightarrow$ $H^{0}\left(\mathcal{O}_{Y}(t)\right)$ is surjective. Hence $h^{1}\left(\mathcal{I}_{Y}(t)\right) \leq h^{1}\left(\mathcal{I}_{X}(t)\right)$. If $X=A \sqcup Z$ and $Y=A \sqcup W$ with $Z$ zero-dimensional, then the restriction map $H^{0}\left(\mathcal{O}_{X}(t)\right) \rightarrow$ $H^{0}\left(\mathcal{O}_{Y}(t)\right)$ is surjective. Hence $h^{1}\left(\mathcal{I}_{Y}(t)\right) \leq h^{1}\left(\mathcal{I}_{X}(t)\right)$.

\section{Low degrees}

In this section we study the triples $(t, a, c)$ whose associated integer $k$ is low.

Lemma 4. We have $h^{0}\left(\mathcal{I}_{X(0,0,2)}(3)\right)=h^{1}\left(\mathcal{I}_{X(0,0,2)}(3)\right)=0$.

Proof. Since $h^{0}\left(\mathcal{O}_{X(0,0,2)}(3)\right)=2 \cdot 10=\left(\begin{array}{l}6 \\ 3\end{array}\right)$, then $h^{1}\left(\mathcal{I}_{X(0,0,2)}(3)\right)=h^{1}\left(\mathcal{I}_{X(0,0,2)}(3)\right)$. Let $Q^{\prime}$ be any smooth quadric surface containing the two lines of $X(0,0,2)_{\text {red }}$, say as members of the ruling $\left|\mathcal{O}_{Q^{\prime}}(1,0)\right|$. Since $X(0,0,2) \cap Q^{\prime} \in\left|\mathcal{O}_{Q^{\prime}}(4,0)\right|$, we have $h^{0}\left(Q^{\prime}, \mathcal{I}_{Q^{\prime} \cap X(0,0,2)}(3)\right)=0$. Hence $Q^{\prime}$ is a component of every cubic 
surface containing $X(0,0,2)$. Since there are infinitely many surfaces $Q^{\prime}$, we get $h^{0}\left(\mathcal{I}_{X(0,0,2)}(3)\right)=0$.

Lemma 5. We have $h^{1}\left(\mathcal{I}_{X(0,1,1)}(3)\right)=0$ and $h^{0}\left(\mathcal{I}_{X(0,1,1)}(3)\right)=6$.

Proof. Write $X(0,1,1)=2 P \sqcup 2 L$ with $L$ a line. Let $H$ be the plane spanned by $L$ and $P$. We have $\operatorname{Res}_{H}(2 L \cup 2 P)=L \cup\{P\}$ and hence

$$
h^{0}\left(\mathcal{I}_{\operatorname{Res}_{H}(X(0,1,1)}(2)\right)=6
$$

and

$$
h^{1}\left(\mathcal{I}_{\operatorname{Res}_{H}(X(0,1,1)}(2)\right)=0 .
$$

Since $h^{0}\left(H, \mathcal{I}_{X(0,1,1) \cap H}(3)\right)=0$, the Castelnuovo's sequence gives the lemma.

Lemma 6. We have $h^{1}\left(\mathcal{I}_{X(1,1,1)}(3)\right)=1$ and $h^{0}\left(\mathcal{I}_{X(1,1,1)}(3)\right)=3$.

Proof. Since $h^{0}\left(\mathcal{O}_{X(1,1,1)}(3)\right)=18$, we have

$$
h^{0}\left(\mathcal{I}_{X(1,1,1)}(3)\right)=2+h^{1}\left(\mathcal{I}_{X(1,1,1)}(3)\right) .
$$

Write $X(1,1,1)=R \sqcup 2 P \sqcup 2 L$ with $P$ a point and $L, R$ lines. Let $H$ be the plane spanned by $R$ and $P$. We have $\operatorname{Res}_{H}(X(1,1,1))=L \cup R \cup\{P\}$. Hence $h^{0}\left(\mathcal{I}_{\operatorname{Res}_{H}(X(1,1,1))}(2)\right)=3$. Since $P \notin R$ and $L \cap R=1, X(1,1,1) \cap H$ is the disjoint union of a multiple conic, the 2-point $\{2 P, H\}$ of $H$ and the point $R \cap H$. Hence $h^{0}\left(H, \mathcal{I}_{X(1,1,1) \cap H}(3)\right)=0$. The Castelnuovo's sequence gives $h^{0}\left(\mathcal{I}_{X(1,1,1)}(3)\right)=3$.

Lemma 7. We have $h^{0}\left(\mathcal{I}_{X(0,2,1)}(3)\right)=2$ and $h^{1}\left(\mathcal{I}_{X(0,2,1)}(3)\right)=0$

Proof. Write $X(0,2,1)=2 L \sqcup 2 O \sqcup 2 P$ with $L$ a line. Let $H$ (resp. $M$ ) be the plane spanned by $L$ and $O$ (resp. $L$ and $P$ ). Every cubic surface containing $2 L \cup 2 O \cup 2 P$ contains $H \cup M$. Since $\operatorname{Res}_{H \cup M}(X(0,2,1))=\{O, P\}$, we have $h^{0}\left(\mathcal{I}_{\operatorname{Res}_{H \cup M}(X(0,2,1))}(1)\right)=2$ and $h^{1}\left(\mathcal{I}_{\operatorname{Res}_{H \cup M}(X(0,2,1))}(1)\right)=0$. Apply the Castelnuovo's sequence.

Lemma 8. We have $h^{0}\left(\mathcal{I}_{X(2,0,1)}(3)\right)=2$ and $h^{1}\left(\mathcal{I}_{X(2,0,1)}(3)\right)=0$

Proof. Let $Q^{\prime}$ be the only quadric containing the 3 disjoint lines $X(2,0,1)_{\text {red }}$, say as a divisor of type $(3,0)$. Since $Q^{\prime} \cap X(2,0,1)$ is a divisor of type $(4,0)$, we have $h^{i}\left(Q^{\prime}, \mathcal{I}_{X(2,0,1) \cap Q^{\prime}}(3)\right)=0, i=0,1$. Since $\operatorname{Res}_{Q^{\prime}}(X(2,0,1))$ is a line, we have $h^{0}\left(\mathcal{I}_{\operatorname{Res}_{Q^{\prime}}(X(2,0,1))}(1)\right)=2$ and $h^{1}\left(\mathcal{I}_{\operatorname{Res}_{Q^{\prime}}(X(2,0,1))}(1)\right)=0$. Apply the Castelnuovo's sequence. 
In the same way we check the following lemma.

Lemma 9. We have $h^{0}\left(\mathcal{I}_{X(t, a, c)}(3)\right)=0$ if $t \geq t^{\prime}, a \geq a^{\prime}, c \geq c^{\prime}$ and $\left(t^{\prime}, a^{\prime}, c^{\prime}\right)$ is one of the following triples: $(0,0,2),(0,3,1),(2,1,1),(3,0,1)$, $(1,2,1)$.

Lemma 10. We have $h^{1}\left(\mathcal{I}_{X(4,0,1)}(4)\right)=0$ and $h^{0}\left(\mathcal{I}_{X(4,0,1)}(4)\right)=2$.

Proof. Since $h^{0}\left(\mathcal{O}_{X(4,0,1)}(4)\right)=33=\left(\begin{array}{l}7 \\ 3\end{array}\right)-2$, we have $h^{0}\left(\mathcal{I}_{X(4,0,1)}(4)\right)=$ $2+h^{1}\left(\mathcal{I}_{X(4,0,1)}(4)\right)$. Take $L, M, R \in\left|\mathcal{O}_{Q}(1,0)\right|$ with $L \neq M, M \neq R$ and $L \neq R$. Take a general $Y \in L(2,0,0)$ and set $X:=Y \cup 2 L \cup M \cup R$. It is sufficient to prove that $h^{1}\left(\mathcal{I}_{X}(4)\right)=0$. Since $Y \cap Q$ is a general union of 4 points, we have $h^{1}\left(Q, \mathcal{I}_{X \cap Q}(4)\right)=h^{1}\left(Q, \mathcal{I}_{Y \cap Q}(0,4)\right)=0$. Since $\operatorname{Res}_{Q}(X)=Y \cup L$ and $h^{1}\left(\mathcal{I}_{Y \cup L}(2)\right)=0$, Castelnuovo's sequence gives $h^{1}\left(\mathcal{I}_{X(4,0,1)}(4)\right)=0$

Since any two points of $\mathbb{P}^{3}$ are contained in a line, Lemma 10 implies the following result.

Lemma 11. We have $h^{0}\left(\mathcal{I}_{X(5,0,1)}(4)\right)=0$.

Lemma 12. We have $h^{0}\left(\mathcal{I}_{X(4,1,1)}(4)\right)=0$ and $h^{1}\left(\mathcal{I}_{X(4,1,1)}(x)\right)=0$ for all $x \geq 5$.

Proof. Take $L, M, R \in\left|\mathcal{O}_{Q}(1,0)\right|$ with $L \neq M, M \neq R$ and $L \neq R$ and a general $O \in Q$. Take a general $Y \in L(2,0,0)$ and set $X:=Y \cup 2 L \cup M \cup R \cup 2 O$. It is sufficient to prove that $h^{0}\left(\mathcal{I}_{X}(4)\right)=0$ and $h^{1}\left(\mathcal{I}_{X}(x)\right)=0$ for all $x \geq 5$. Since $Y \cap Q$ is a general union of 4 points, we have $h^{1}\left(Q, \mathcal{I}_{X \cap Q}(4)\right)=0$ and $h^{1}\left(Q, \mathcal{I}_{X \cap Q}(y)\right)=0$ for all $y \geq 5$. Since $\operatorname{Res}_{Q}(X)=Y \cup L \cup\{O\}, Y \nsubseteq Q$ and $O$ is general in $Q$, we have $h^{0}\left(\mathcal{I}_{\operatorname{Res}_{Q}(X)}(2)\right)=0$ and $h^{1}\left(\mathcal{I}_{\operatorname{Res}_{Q}(X)}(x-2)\right)=0$ for all $x \geq 5$. Apply the Castelnuovo's sequence.

Lemma 13. We have $h^{1}\left(\mathcal{I}_{X(3,1,1)}(4)\right)=h^{0}\left(\mathcal{I}_{X(3,2,1)}(4)\right)=0$, while we have $h^{1}\left(\mathcal{I}_{X(3,2,1)}(x)\right)=0$ for all $x \geq 5$.

Proof. Fix $L, M, R \in\left|\mathcal{O}_{Q}(1,0)\right|$ with $L \neq M, M \neq R$ and $L \neq R$. Take a general $Y \in L(1,1,0)$ and set $X:=2 L \cup M \cup R \in L(3,1,1)$. We have $\operatorname{Res}_{Q}(X)=$ $Y, h^{1}\left(\mathcal{I}_{Y}(2)\right)=0$ and $h^{1}\left(Q, \mathcal{I}_{X \cap Q}(4)\right)=0$, proving the first equality. Fix a plane $H$, a line $L \subset H, O \in H \backslash L$, and a general $Y \in L(3,1,0)$. Set $X^{\prime}:=Y \cup 2 L \cup 2 O$. We have $\operatorname{Res}_{H}\left(X^{\prime}\right)=Y \cup\{O\} \cup L$. Since $Y \cup L$ is general in $L(4,1,0)$, we have $h^{i}\left(\mathcal{I}_{Y \cup L}(3)\right)=0, i=0,1$ (Lemma 1) and hence $h^{0}\left(\mathcal{I}_{\operatorname{Res}_{H}\left(X^{\prime}\right)}(3)\right)=0$ and $h^{1}\left(\mathcal{I}_{\operatorname{Res}_{H}\left(X^{\prime}\right)}(x-1)\right)=0$ for all $x \geq 5$. Use the Castelnuovo's sequence. 
Lemma 14. We have $h^{0}\left(\mathcal{I}_{X(2,3,1)}(4)\right)=h^{1}\left(\mathcal{I}_{X(2,3,1)}(4)\right)=1$, while we have $h^{1}\left(\mathcal{I}_{X(2,3,1)}(x)\right)=0$ for all $x \geq 5$.

Proof. Since $h^{0}\left(\mathcal{I}_{X(2,3,1)}(4)\right)=\left(\begin{array}{l}7 \\ 3\end{array}\right)$, we have

$$
h^{0}\left(\mathcal{I}_{X(2,3,1)}(4)\right)=h^{1}\left(\mathcal{I}_{X(2,3,1)}(4)\right) .
$$

Write $X(2,3,1)=2 L \sqcup M \sqcup R \sqcup 2 S$ with $\sharp(S)=3$. Let $Q_{1}$ (resp. $Q_{2}$ ) be the only quadric containing $L \cup M \cup S$ (resp. $L \cup R \cup S$ ). Since $X(2,3,1) \subset$ $Q_{1} \cup Q_{2}$, we have $h^{0}\left(\mathcal{I}_{X(2,3,1)}(4)\right) \geq 1$. Fix a plane $H$, a line $R \subset H$, general $P_{1}, P_{2} \in H$ and a general $Y \in L(1,1,1)$. Set $X:=Y \cup R \cup 2 P_{1} \cup 2 P_{2}$. We have $h^{i}\left(H, \mathcal{I}_{X \cap H}(4)\right)=0, i=0,1$, and $\operatorname{Res}_{H}(X)=Y \cup\left\{P_{1}, P_{2}\right\}$. We have $h^{1}\left(\mathcal{I}_{Y}(3)\right)=1$ and $h^{0}\left(\mathcal{I}_{Y}(3)\right)=3$ (Lemma 6). Since $h^{0}\left(\mathcal{I}_{Y}(3)\right)<4$, we have $h^{0}\left(\mathcal{I}_{Y}(2)\right)=0$. Lemma 3 gives $h^{0}\left(\mathcal{I}_{Y \cup\left\{P_{1}, P_{2}\right\}}(3)\right)=1$. Apply the Castelnuovo's sequence. Since $h^{1}\left(\mathcal{I}_{Y \cup\left\{P_{1}, P_{2}\right\}}(4)\right)=0$, we also get $h^{1}\left(\mathcal{I}_{X}(x)\right)=0$ for all $x \geq$ 5.

Lemma 15. We have $h^{0}\left(\mathcal{I}_{X(0,5,1)}(4)\right)=3$ and $h^{1}\left(\mathcal{I}_{X(0,5,1)}(4)\right)=1$. We have $h^{1}\left(\mathcal{I}_{X(1,5,1)}(x)\right)=0$ for all $x \geq 5$.

Proof. Since $h^{0}\left(\mathcal{O}_{X(0,5,1)}(4)\right)=33, h^{0}\left(\mathcal{I}_{X(0,5,1)}(4)\right)=2+h^{1}\left(\mathcal{I}_{X(0,5,1)}(4)\right)$. Set $D:=X(0,5,1)_{\text {red }}$. We have $h^{0}\left(\mathcal{I}_{D}(2)\right)=2$. Fix any $Q_{1}, Q_{2} \in\left|\mathcal{I}_{D}(2)\right|$. Since $X(3,0,1) \subset Q_{1} \cup Q_{2}$, we get $h^{0}\left(\mathcal{I}_{X(0,5,1)}(4)\right) \geq 3$. Fix any smooth $Q \in$ $\left|\mathcal{I}_{D}(2)\right|$. Since $h^{0}\left(Q, \mathcal{I}_{Z}(2,4)\right)=h^{1}\left(Q, \mathcal{I}_{Z}(2,4)\right)=1$ for a general union $Z \subset$ $Q$ of five 2-points of $Q$ (this is one of the exceptional cases in [14, Table I], $\operatorname{Res}_{Q}(X(0,5,1))=D$ and $h^{1}\left(\mathcal{I}_{D}(2)\right)=0$, we get the first two assertions. Since $h^{1}\left(Q, \mathcal{I}_{Z}(y-2, y)\right)=0$ for all $y \geq 5$, we get $h^{1}\left(\mathcal{I}_{X(1,5,1)}(x)\right)=0$ for all $x \geq 5$.

Lemma 16. We have $h^{0}\left(\mathcal{I}_{X(1,5,1)}(4)\right)=0$ and $h^{1}\left(\mathcal{I}_{X(1,5,1)}(x)\right)=0$ for all $x \geq 5$.

Proof. Write $X(1,5,1)=R \cup 2 L \cup 2 S$ with $S=\left\{P_{1}, P_{2}, P_{3}, P_{4}, P_{5}\right\}$ general points of $\mathbb{P}^{3}$. Fix a smooth quadric surface $Q \in\left|\mathcal{I}_{S \cup L}(2)\right|$. Since $h^{0}\left(Q, \mathcal{I}_{Z}(2,4)\right)=$ 1 and $h^{1}\left(Q, \mathcal{I}_{Z}(3,5)\right)=0$ for a general union $Z \subset Q$ of 5 2-points of $Q$ and $R \cap Q$ is a general union of 2 points of $Q$, we get $h^{0}\left(Q, \mathcal{I}_{X(1,5,1)}(4)\right)=0$ and $h^{1}\left(Q, \mathcal{I}_{X(1,5,1)}(x)\right)=0$ for all $x \geq 5$. Since $\operatorname{Res}_{Q}(X(1,5,1))=L \cup R \cup S$ is contained in no quadric surface, a Castelnuovo's sequence gives the lemma.

Lemma 17. We have $h^{0}\left(\mathcal{I}_{X(0,6,1)}(4)\right)=1$ and $h^{1}\left(\mathcal{I}_{X(0,6,1)}(4)\right)=3$. We have $h^{1}\left(\mathcal{I}_{X(0,6,1)}(x)\right)=0$ for all $x \geq 5$. 
Proof. Set $L \cup S:=X(0,6,1)_{\text {red }}$ with $L$ the line. Let $Q$ be the only quadric containing $L \cup S$. $Q$ is smooth. We have $\operatorname{Res}_{Q}(2 S \cup 2 L)=S \cup L$. We have $h^{0}\left(Q, \mathcal{I}_{X(0,6,1) \cap Q}(4)\right)=h^{0}\left(Q, \mathcal{I}_{(2 S \cap Q)}(2,4)\right)=0$. Hence the Castelnuovo's sequence gives $h^{0}\left(\mathcal{I}_{X(0,6,1)}(4)\right)=h^{0}\left(\mathcal{I}_{L \cup S}(2)\right)=1$. Since $h^{0}\left(\mathcal{I}_{X(0,6,1)}(4)\right)=37$, we have $h^{1}\left(\mathcal{I}_{X(0,6,1)}(4)\right)=2+h^{0}\left(\mathcal{I}_{X(0,6,1)}(4)\right)=0$. Since $h^{1}\left(Q, \mathcal{I}_{(2 S \cap Q)}(3,5)\right)=$ 0 , the Castelnuovo's sequence gives $h^{1}\left(\mathcal{I}_{X(0,6,1)}(x)\right)=0$ for all $x \geq 5$.

Lemma 18. We have $h^{0}\left(\mathcal{I}_{X(1,1,2)}(4)\right)=h^{1}\left(\mathcal{I}_{X(1,1,2)}(4)\right)=1$, while we have $h^{1}\left(\mathcal{I}_{X(1,1,2)}(x)\right)=0$ for all $x \geq 5$.

Proof. Since $h^{0}\left(\mathcal{O}_{X(1,1,2)}(4)\right)=35$, we have $h^{0}\left(\mathcal{I}_{X(1,1,2)}(4)\right)=h^{1}\left(\mathcal{I}_{X(1,1,2)}(4)\right)$. Write $X(1,1,2)=Z \sqcup 2 L \sqcup 2 R \sqcup M$ with $L, M, N$ general lines. Let $Q$ be the only cubic containing $L \cup R \cup M$, say as a divisor of type (3,0). Since $Z$ is general, we have $Z_{\text {red }} \notin Q$, i.e. $\operatorname{Res}_{Q}(X(1,1,2))=L \cup R \cup Z$. Since $h^{0}\left(Q, \mathcal{I}_{X(1,1,2) \cap Q}(4)\right)=$ 0 , we have $h^{0}\left(\mathcal{I}_{X(1,1,2)}(4)\right)=h^{0}\left(\mathcal{I}_{Z \cup L \cup R}(2)\right)$. Set $\{P\}:=Z_{\text {red }}$. The only quadric surface containing $Z \cup L \cup R$ is the union of the two planes through $P$ containing one of the lines $L, R$. Since $h^{1}\left(Q, \mathcal{I}_{Q \cap X(1,1,2)}(t)\right)=0$ and $h^{1}\left(\mathcal{I}_{Z \cup L \cup R}(x-\right.$ $2))=0$ for all $x \geq 5$, we get the last assertion.

Lemma 19. We have $h^{0}\left(\mathcal{I}_{X(2,0,2)}(4)\right)=1$ and $h^{1}\left(\mathcal{I}_{X(2,0,2)}(4)\right)=2$. We have $h^{1}\left(\mathcal{I}_{X(2,0,2)}(x)\right)=0$ for all $x \geq 5$.

Proof. Write $X(2,0,2)=L \sqcup R \sqcup 2 M \sqcup 2 N$. Let $Q_{1}$ (resp. $Q_{2}$ ) be the only quadric containing $L \cup M \cup N$ (resp. $N \cup M \cup N$ ), say as a curve of type $(3,0)$ (each $Q_{i}$ is smooth). Since each curve $Q_{i} \cap X(2,0,2)$ has type $(5,0)$, no curve of type $(4,4)$ contains it. Hence $Q_{1} \cup Q_{2}$ is the only quartic surface containing $X(2,0,2)$. Since $h^{0}\left(\mathcal{O}_{X(2,0,2)}(4)\right)=36$, we have $h^{1}\left(\mathcal{I}_{X(2,0,2)}(4)\right)=$ $h^{0}\left(\mathcal{I}_{X(2,0,2)}(4)\right)+1$. Since $h^{1}\left(Q_{1}, \mathcal{I}_{X(2,0,2) \cap Q_{1}}(5)\right)=0$, we get $h^{1}\left(\mathcal{I}_{X(2,0,2)}(x)\right)=$ 0 for all $x \geq 5$.

Lemma 20. We have $h^{0}\left(\mathcal{I}_{X(0,2,2)}(4)\right)=3$ and $h^{1}\left(\mathcal{I}_{X(0,2,2)}(4)\right)=2$.

Proof. Write $X(0,2,2)=2 O \sqcup 2 P \sqcup 2 M \sqcup 2 N$ with $M, N$ lines and $O, P$ points. Since $M, N, O$, and $P$ are general, we have $h^{0}\left(\mathcal{I}_{\{O, P\} \cup M \cup N}(2)\right)=2$. Fix any $Q_{1}, Q_{2} \in\left|\mathcal{I}_{\{O, P\} \cup M \cup N}(2)\right|$. Since $\left.Q_{1} \cup Q_{2} \in \mid \mathcal{I}_{X(0,2,2)}(4)\right)$, we get $h^{0}\left(\mathcal{I}_{X(0,2,2)}(4)\right) \geq 3$. To check the opposite inequality fix a smooth $Q^{\prime} \in$ $\left|\mathcal{I}_{\{O, P\} \cup M \cup N}(2)\right|$, a general $P_{1} \in Q^{\prime}$ and general points $P_{2}, P_{3} \in \mathbb{P}^{3}$. We have $h^{0}\left(Q^{\prime}, \mathcal{I}_{\left(X(0,2,2) \cap Q^{\prime}\right) \cup\left\{P_{1}\right\}}(4)\right)=0$, because $h^{0}\left(Q^{\prime}, \mathcal{I}_{\left\{2 O, Q^{\prime}\right\} \cup\left\{2 P, Q^{\prime}\right\}}(0,4)\right)=1$. Since $\left\{P_{2}, P_{3}\right\}$ are general in $\mathbb{P}^{3}$, we have $h^{0}\left(\mathcal{I}_{\left\{P_{2}, P_{3}, O, P\right\} \cup M \cup N}(2)\right)=0$. Since $\operatorname{Res}_{Q^{\prime}}\left(\left\{P_{1}, P_{2}, P_{3}\right\} \cup X(0,2,2)\right)=\left\{P_{2}, P_{3}, O, P\right\} \cup M \cup N$, the Castelnuovo's sequence gives $h^{0} 0\left(\mathcal{I}_{X(0,2,2)}(4)\right) \leq 3$. 
Since $h^{0}\left(\mathcal{O}_{X(0,2,2)}(4)\right)=34$, we have $h^{1}\left(\mathcal{I}_{X(0,2,2)}(4)\right)=h^{0}\left(\mathcal{I}_{X(0,2,2)}(4)\right)-$ 1. 0 .

Lemma 21. We have $h^{0}\left(\mathcal{I}_{X(3,0,2)}(4)\right)=h^{0}\left(\mathcal{I}_{X(2,1,2)}(4)\right)=h^{0}\left(\mathcal{I}_{X(1,2,2)}(4)\right)=$

Proof. Since a line contains two points and a 2-point contains a point, Lemma 19 implies $h^{0}\left(\mathcal{I}_{X(3,0,2)}(4)\right)=h^{0}\left(\mathcal{I}_{X(2,1,0)}(4)\right)=0$. Write $X(1,2,2)=$ $L \sqcup 2 N \sqcup 2 M \sqcup 2 P \sqcup 2 O$ and call $H$ the plane spanned by $N$ and $P$. The scheme $X(1,2,2) \cap H$ is the union of an unreduced conic, two general 2-points of $H$ (one of them being $M \cap H$ ) and a general point (i.e. $L \cap H$ ). Hence $h^{0}\left(H, \mathcal{I}_{X(1,2,2) \cap H}(4)\right)=0$. The scheme $\operatorname{Res}_{H}(X(1,2,2))$ is a general union of a general element of $L(1,1,1)$ and a general point, $O$, of $H$. Lemmas 3 and 6 and a Castelnuovo's sequence gives $h^{0}\left(\mathcal{I}_{X(1,2,2)}(4)\right)=0$; alternatively, use Lemma 18.

Lemma 22. We have $h^{0}\left(\mathcal{I}_{X(0,3,2)}(4)\right)=1$ and $h^{1}\left(\mathcal{I}_{X(0,3,2)}(4)\right)=4$.

Proof. Write $X(0,3,2)=2 P_{1} \sqcup 2 P_{2} \sqcup 2 P_{3} \sqcup L \sqcup 2 M$ with $L, M$ lines and $P_{1}, P_{2}, P_{3}$ general points. Let $Q^{\prime}$ be the only quadric surface containing $\left\{P_{1}, P_{1}, P_{3}\right\} \cup L \cup M$. Since $2 Q^{\prime}$ is a quartic surface containing $X(0,3,2)$, we have $h^{0}\left(\mathcal{I}_{X(0,3,2)}(4)\right) \geq 1$. The quadric $Q^{\prime}$ is smooth. Call $\mathcal{O}_{Q^{\prime}}(1,0)$ the ruling of $Q^{\prime}$ containing $L$. The singular points of any $D \in\left|\mathcal{O}_{Q^{\prime}}(0, e)\right|$, $e>0$, are the union of the multiple components of $D$. Since $X(0,3,2) \cap Q^{\prime}$ is the union of a divisor of type $(4,0)$ and3 general 2-points of $Q^{\prime}$, we have $h^{0}\left(Q^{\prime}, \mathcal{I}_{X(0,3,2) \cap Q^{\prime}}(4)\right)=0$. Since $Q^{\prime}$ is the only quadric surface containing $\left\{P_{1}, P_{1}, P_{3}\right\} \cup L \cup M=\operatorname{Res}_{Q^{\prime}}(X(0,3,2))$, the Castelnuovo's sequence gives $h^{0}\left(\mathcal{I}_{X(0,3,2)}(4)\right) \leq 1$. Since $h^{0}\left(\mathcal{O}_{X(0,3,2)}(4)\right)=38$, we have $h^{1}\left(\mathcal{I}_{X(0,3,2)}(4)\right)=$ $h^{0}\left(\mathcal{I}_{X(0,3,2)}(4)\right)+3$.

Lemma 23. We have $h^{0}\left(\mathcal{I}_{X}(4)\right)=1, h^{1}\left(\mathcal{I}_{X}(4)\right)=5$ and $h^{1}\left(\mathcal{I}_{X}(x)\right)=0$ for every $x \geq 5$ and every $X \in L(0,0,3)$.

Proof. Set $D:=X_{\text {red }}$ and call $Q^{\prime}$ the only quadric containing $D . Q^{\prime}$ is smooth and we call $(1,0)$ the ruling of $Q^{\prime}$ such that $X \cap Q^{\prime} \in\left|\mathcal{O}_{Q^{\prime}}(6,0)\right|$. Since any two $D$ 's are projectively equivalent, all $X \in L(0,0,3)$ are projectively equivalent. We have $h^{0}\left(Q^{\prime}, \mathcal{I}_{X \cap Q^{\prime}}(4)\right)=0, h^{1}\left(Q^{\prime}, \mathcal{I}_{X \cap Q^{\prime}}(t)\right)=0$ for all $t \geq 5$, and $\operatorname{Res}_{Q^{\prime}}(X(0,0,3))=D$. The Castelnuovo's sequence gives $h^{0}\left(\mathcal{I}_{X(0,0,3)}(4)\right)=$ $h^{0}\left(\mathcal{I}_{D}(2)\right)=1$. Since $h^{0}\left(\mathcal{O}_{X}(4)\right)=3 \cdot 13=\left(\begin{array}{l}7 \\ 3\end{array}\right)+4$, we have $h^{1}\left(\mathcal{I}_{X(0,0,3)}(4)\right)=$ $h^{0}\left(\mathcal{I}_{X(0,0,3)}(4)\right)+4$. Since $h^{1}\left(\mathcal{I}_{D}(x-2)\right)=0$ for all $x \geq 5$, we get $h^{1}\left(\mathcal{I}_{X}(x)\right)=0$ for all $x \geq 5$. 
Remark 4. By Lemmas 23, 21, and 22 we have $h^{0}\left(\mathcal{I}_{X(t, a, c)}(4)\right)=0$ if $t \geq t^{\prime}, c \geq c^{\prime}, a \geq a^{\prime}$ and $\left(t^{\prime}, a^{\prime}, c^{\prime}\right)$ is one of the following triples: $(1,0,3)$, $(0,1,3),(0,0,4),(3,0,2),(2,1,2),(1,2,2)$.

Lemma 24. We have $h^{0}\left(\mathcal{I}_{X(1,0,3)}(5)\right)=4$ and $h^{1}\left(\mathcal{I}_{X(1,0,3)}(5)\right)=2$. We have $h^{1}\left(\mathcal{I}_{X(1,0,3)}(x)\right)=0$ for all $x \geq 6$.

Proof. Write $X(1,0,3)=L \sqcup A$ with $L$ a line and let $Q^{\prime}$ be the only quadric containing $D:=A_{\text {red }}$, say as an element of $\left|\mathcal{O}_{Q^{\prime}}(3,0)\right|$. Since $A \cap Q^{\prime} \in$ $\left|\mathcal{O}_{Q^{\prime}}(6,0)\right|$, we have $h^{0}\left(Q^{\prime}, \mathcal{I}_{X(1,0,3) \cap Q^{\prime}}(5)\right)=0$. The Castelnuovo's sequence $(1)$ gives $h^{0}\left(\mathcal{I}_{X(1,0,3)}(5)\right)=h^{0}\left(\mathcal{I}_{L \cup D}(3)\right)=4([13])$. Since $h^{0}\left(\mathcal{O}_{X(1,0,3)}(5)\right)=54=$ $\left(\begin{array}{l}8 \\ 3\end{array}\right)-2$, we get $h^{1}\left(\mathcal{I}_{X(1,0,3)}(5)\right)=2$. Since $X(1,0,3) \cap Q^{\prime}$ is the union of $A \cap Q^{\prime}$ and two general points of $Q^{\prime}$, we have $h^{1}\left(Q^{\prime}, \mathcal{I}_{X(1,0,3) \cap Q^{\prime}}(x)\right)=0$ for all $x \geq 6$. The Castelnuovo's sequence $(1)$ gives $h^{1}\left(\mathcal{I}_{X(1,0,3)}(x)\right)=h^{1}\left(\mathcal{I}_{D \cup A}(x)\right)=0$ for all $x \geq 6$.

Lemma 25. We have $h^{0}\left(\mathcal{I}_{X(2,0,3)}(5)\right)=0$ and $h^{1}\left(\mathcal{I}_{X(2,0,3)}(x)\right)=0$ for all $x \geq 6$.

Proof. Write $X(2,0,3)=R \sqcup A$ with $R \in L(2,0,0)$ and $A \in L(0,0,3)$. Let $Q^{\prime}$ be the only quadric surface containing $D:=A_{\text {red }}$, say as an element of $\left|\mathcal{O}_{Q^{\prime}}(3,0)\right|$. Since $X(2,0,3) \cap Q^{\prime}$ is a general union of an element of $\left|\mathcal{O}_{Q^{\prime}}(6,0)\right|$ and 4 general points of $Q^{\prime}$, the Castelnuovo's sequence gives $h^{0}\left(\mathcal{I}_{X(2,0,3)}(5)\right)=$ $h^{0}\left(\mathcal{I}_{R \cup D}(3)\right)=0$ and $h^{1}\left(\mathcal{I}_{X(2,0,3)}(x)\right)=h^{0}\left(\mathcal{I}_{R \cup D}(x-2)\right)=0$ for all $x \geq 6$ $([13])$.

Lemma 26. We have $h^{0}\left(\mathcal{I}_{X(1,1,3)}(5)\right)=0$ and $h^{1}\left(\mathcal{I}_{X(1,1,3)}(x)\right)=0$ for all $x \geq 6$.

Proof. Write $X(1,1,3)=L \sqcup Z \sqcup A$ with $A \in L(0,0,3)$ and $Z \in L(0,1,0)$. Let $Q^{\prime}$ be the only quadric containing $D:=A_{\text {red }}$, say as a divisor of type $(3,0)$. Since $X(1,1,3)$ is general, then $Z \cap Q^{\prime}=\emptyset$ and $L \cap Q^{\prime}$ is a general union of 2 points of $Q^{\prime}$. Hence $h^{0}\left(\mathcal{I}_{X(1,1,3)}(5)\right)=h^{0}\left(\mathcal{I}_{L \cup D \cup Z}(3)\right)=0$ ([4]) and $h^{1}\left(\mathcal{I}_{X(1,1,3)}(x)\right)=h^{0}\left(\mathcal{I}_{L \cup D \cup Z}(x-2)\right)=0$ for all $x \geq 6$.

Lemma 27. We have $h^{i}\left(\mathcal{I}_{X(0,2,3)}(5)\right)=0, i=0,1$.

Proof. Write $X(0,2,3)=Z \sqcup A$ with $A \in L(0,0,3)$ and $Z \in L(0,2,0)$. Let $Q^{\prime}$ be the only quadric containing $D:=A_{\text {red }}$, say as a divisor of type $(3,0)$. Since $Z$ is general, then $Z \cap Q^{\prime}=\emptyset$. Hence $h^{i}\left(\mathcal{I}_{X(0,2,3)}(5)\right)=h^{i}\left(\mathcal{I}_{D \cup Z}(3)\right)=0$, $i=0,1$ (Lemma 1). 
Lemma 28. We have $h^{1}\left(\mathcal{I}_{X(t, a, 1)}(5)\right)=0$ for all $(a, t) \in \mathbb{N}^{2}$ such that $6 t+4 a+16 \leq 56$.

Proof. Increasing if necessary $a$ we reduce to the case $37 \leq 6 t+4 a \leq 40$. Set $e:=\lfloor(10-t) / 3\rfloor$ and $f:=10-t-3 e$. The quadruples $(t, a, e, f)$ are the following ones: $(6,1,1,1),(5,2,1,2),(4,4,2,0),(3,5,2,1),(2,7,2,2),(1,8,3,0)$, $(0,10,3,1)$. By the semicontinuity theorem it is sufficient to prove the existence of $X \in L(t, a, 1)$ such that $h^{1}\left(\mathcal{I}_{X}(5)\right)=0$.

(i) Assume $t \leq 4$. Let $H \subset \mathbb{P}^{3}$ be a plane. We fix $L \in L(0,0,1)$ contained in $H$ and take a general $S \cup S^{\prime} \subset H$ with $\sharp(S)=e, \sharp\left(S^{\prime}\right)=f$. Set $A:=2 L$. Fix a general $W \in L(t, a-e-f, 0)$. We have $h^{i}\left(H, \mathcal{I}_{E}(3)\right)=0, i=0,1$, for a general union $E \subset H$ of $f+t$ points and $e$ 2-points of $H$. By the differential Horace lemma for double points ([1], [11, Lemma 5], [2] in characteristic $\neq 2$ ) to prove that a general union of $W \cup 2 L \cup 2 S$ and $f$ 2-points (and hence to prove the lemma in these cases) it is sufficient to prove that $h^{1}\left(\mathcal{I}_{W \cup L \cup S \cup\left\{2 S^{\prime}, H\right\}}(4)\right)=0$.

Claim 1: $h^{1}\left(\mathcal{I}_{W \cup L \cup\left\{2 S^{\prime}, H\right\}}(4)\right)=0$.

Proof of Claim 1: If $t \in\{4,1\}$, then $S^{\prime}=\emptyset$ and hence Claim 1 is true by [4]. Now assume $t \in\{0,3\}$. In this case $S^{\prime}$ is a point. Since any union of a line and a point is contained in a plane, $W \cup L \cup 2 S^{\prime}$ may be considered as a general element of $L(t+1, a-e+1,0)$. In these two cases we have $h^{1}\left(\mathcal{I}_{W \cup L \cup 2 S^{\prime}}(4)\right)=0$ by [4], Hence $h^{1}\left(\mathcal{I}_{W \cup L \cup\left\{2 S^{\prime}, H\right\}}(4)\right)=0$ (Lemma 3 ).

Now assume $(t, a, e, f)=(2,7,2,2)$. Since $W \cap H$ is a general subset with cardinality 2 , we have $h^{1}\left(H, \mathcal{I}_{H \cap\left(W \cup L \cup S \cup\left\{2 S^{\prime}, H\right\}\right)}(4)\right)=0$. Hence by the Castelnuovo's sequence it is sufficient to use that $h^{1}\left(\mathcal{I}_{W}(3)\right)=0$ in this case, because $W \in L(3,2,0)$ (Lemma 1).

Claim 1 implies $h^{0}\left(\mathcal{I}_{W \cup L \cup\left\{2 S^{\prime}, H\right\}}(4)\right)=e+40-4 a-6 t$. Since $S$ is general in $H$ and $\operatorname{Res}_{H}\left(W \cup L \cup\left\{2 S^{\prime}, H\right\}\right)=W$, to get $h^{1}\left(\mathcal{I}_{W \cup L \cup S \cup\left\{2 S^{\prime}, H\right\}}(4)\right)=0$ it is sufficient to prove that $h^{0}\left(\mathcal{I}_{W}(3)\right) \leq 40-6 t-4 a$. We have $h^{0}\left(\mathcal{O}_{W}(3)\right)=$ $4 t+4(a-e-f)$; we use Lemma 1 , because $W \notin L(2,3,0)$.

(ii) Now assume $t \in\{5,6\}$. First assume $(t, a)=(6,1)$. Let $Z \subset \mathbb{P}^{3}$ be a general 2-point. Take $L \in\left|\mathcal{O}_{Q}(1,0)\right|$ and $F \in\left|\mathcal{O}_{Q}(3,0)\right|$ such that $F$ is reduced and $L \cap F=\emptyset$. Set $A:=2 L$, so that $A \cap Q \in\left|\mathcal{O}_{Q}(2,0)\right|$ and $\operatorname{Res}_{Q}(A)=L$. Set $W:=G \cup F \cup A$. Since $G$ is general, $Q \cap G$ is a general union of 6 points. Hence $h^{i}\left(Q, \mathcal{I}_{W \cap Q}(5)\right)=0, i=0,1$. Hence by the Castelnuovo's sequence it is sufficient to use that $h^{i}\left(\mathcal{I}_{Z \cup L \cup G}(3)\right)=0, i=0,1$ by [4]. Now assume $(t, a)=(5,2)$. Take $L, A=2 L$ and $F$ as in the previous case. Fix a general $G^{\prime} \in L(2,0,0)$ and a general 2-point $Z^{\prime}$ of $\mathbb{P}^{3}$. Set $Z:=Z^{\prime} \cup 2 O$. By the semicontinuity theorem it is sufficient to prove that $h^{1}\left(\mathcal{I}_{Z \cup G^{\prime} \cup A \cup F}(5)\right)=0$. Since $\left(G^{\prime} \cup Z\right) \cap Q$ is a general union of 4 points and one 2-point of $Q$, we 
have $h^{i}\left(Q, \mathcal{I}_{Q \cap\left(Z \cup G^{\prime} \cup A \cup F\right)}(5)\right)=0$. Hence by the Castelnuovo's sequence it is sufficient to prove that $h^{1}\left(\mathcal{I}_{G^{\prime} \cup Z^{\prime} \cup\{O\} \cup L}(3)\right)=0$. We have $h^{1}\left(\mathcal{I}_{G^{\prime} \cup L \cup Z^{\prime}}(3)\right)=0$ ([4]) and hence $h^{0}\left(\mathcal{I}_{G^{\prime} \cup L \cup Z^{\prime}}(3)\right)=3$. Since $h^{0}\left(\mathcal{I}_{G^{\prime} \cup Z}(1)\right)=0$ and $O$ is general in $Q$, we get $h^{0}\left(\mathcal{I}_{G^{\prime} \cup L \cup Z^{\prime} \cup\{O\}}(3)\right)=2$. and hence $h^{1}\left(\mathcal{I}_{G^{\prime} \cup L \cup Z^{\prime} \cup\{O\}}(3)\right)=0$.

(iii) Assume $(t, a)=(2,7)$. We take $R, L, M \in\left|\mathcal{O}_{Q}(1,0)\right|$ with $L \neq R$, $L \neq M$ and $R \neq M$. We take a general $S \subset Q$ with $\sharp(S)=4$ and a general $Z \in L(0,3,0)$. Set $X:=2 L \cup R \cup M \cup 2 S \cup Z$. It is sufficient to prove that $h^{1}\left(\mathcal{I}_{X}(5)\right)=0$. Since $X \cap Q$ is the union of an element of $\left|\mathcal{O}_{Q}(4,0)\right|$ and 4 general 2-points of $Q$, we have $h^{i}\left(Q, \mathcal{I}_{X \cap Q}(5)\right)=0, i=0,1$ ([14, Proposition 5.2 and Theorem 7.2]). Hence by the Castelnuovo's sequence it is sufficient to prove that $h^{1}\left(\mathcal{I}_{Z \cup S \cup L}(3)\right)=0$. We have $h^{1}\left(\mathcal{I}_{Z \cup L}(3)\right)=0$ and $h^{0}\left(\mathcal{I}_{Z \cup L}(4)\right)=4($ Lemma 1$)$. Since $S$ is general in $Q$ and $h^{0}\left(\mathcal{I}_{Z}(1)\right)=0$, Lemma 3 gives $h^{i}\left(\mathcal{I}_{\operatorname{Res}_{Q}(X)}(3)\right)=0$, $i=0,1$.

Lemma 29. We have $h^{1}\left(\mathcal{I}_{X(t, a, 2)}(5)\right)=0$ for all $(a, t) \in \mathbb{N}^{2}$ such that $6 t+4 a+32 \leq 56$.

Proof. Set $\beta:=24-6 t-4 a$. Increasing if necessary $a$ we reduce to the case $0 \leq 6 t+4 a \leq 3$. Hence it is sufficient to check the following triples $(t, a, \beta)$ : $(4,0,0),(3,1,2),(2,3,0),(1,4,2),(0,6,0)$.

(i) First assume $3 \leq t \leq 4$. Fix $L, R, M \in\left|\mathcal{O}_{Q}(1,0)\right|$ such that $R \neq L$, $R \neq M$ and $L \neq M$. Fix a general $W \in L(t-1, a, 0)$ and set $X:=W \cup 2 L \cup 2 R \cup$ $M$. Since $W \cap Q$ is a general union of $2 t-2$ points and $(2 L \cup 2 R \cup M) \in\left|\mathcal{O}_{Q}(5,0)\right|$, we have $h^{1}\left(Q, \mathcal{I}_{X \cap Q}(5)\right)=0$. By the Castelnuovo's sequence it is sufficient to use that $h^{1}\left(\mathcal{I}_{W \cup L}(3)\right)=0$ by Lemma 1 .

(ii) Assume $(t, a)=(2,3)$. Fix a plane $H$, a general line $R$, a line $L \subset H$, a general $Y \in L(2,0,0)$, a general $O \in H$ and a general $B \subset H$ such that $\sharp(B)=2$. Set $X^{\prime}:=2 L \sqcup 2 R \sqcup 2 O \sqcup Y$. We have $h^{i}\left(H, \mathcal{I}_{\left(X^{\prime} \cap H\right) \cup S^{\prime}}(6)\right)=0$, $i=0,1$, and $\operatorname{Res}_{H}\left(X^{\prime}\right)=2 R \cup L \cup Y \cup\{O\}$. By the differential Horace lemma for 2-points ([1], [11, Lemma 5], [2] in characteristic $\neq 2$ ) to prove that a general union $X$ of $X^{\prime}$ and two general 2-points of $\mathbb{P}^{3}$ satisfies $h^{i}\left(\mathcal{I}_{X}(5)\right)=0$, $i=0,1$, (and hence to prove the lemma for the pair $(t, a)=(2,3))$ it is sufficient to prove that $h^{i}\left(\mathcal{I}_{\{2 B, H\} \cup 2 R \cup L \cup Y \cup\{O\}}(4)\right)=0, i=0,1$. Let $M \subset \mathbb{P}^{3}$ be a general plane containing $R$. Set $D:=H \cap M$. Write $B=\left\{P_{1}, P_{2}\right\}$ with $P_{1}, P_{2}$ general points of $H$. We specialize $O$ to a general point $O_{1}$ of $D$. We specialize $\left\{2 P_{1}, H\right\} \cup\left\{2 P_{2}, H\right\}$ to $\left\{2 P_{1}, H\right\} \cup\left\{2 O^{\prime}, H\right\}$ with $O^{\prime}$ general point of $D$. Set $Y^{\prime \prime}:=\left\{2 P_{1}, H\right\} \cup\left\{2 O^{\prime}, H\right\} \cup 2 R \cup L \cup Y \cup\left\{O_{1}\right\}=Y_{1} \sqcup 2 R$. We have $\left\{2 O^{\prime}, H\right\} \cap M=2 O^{\prime} \cap D$, i.e. $\left\{2 O^{\prime}, H\right\} \cap M$ is the degree two scheme with $O^{\prime}$ as its reduction and contained in $D$. We get $h^{i}\left(M, \mathcal{I}_{Y_{1} \cap M}(2)\right)=0, i=0,2$, because $(Y \cup L) \cap M$ is a general union of $\left\{2 O^{\prime}, H\right\} \cap M$, the general point $O_{1}$ 
of $D$ and 3 general points of $M$. Since $h^{i}\left(\mathcal{I}_{\operatorname{Res}_{M}\left(Y^{\prime}\right)}(3)\right)=0, i=0,1$, we are done.

(iii) Take $(t, a, \beta)=(1,4,2)$. Take $L, R, M \in\left|\mathcal{O}_{Q}(1,0)\right|$ with $L \neq R$, $L \neq M$ and $R \neq M$. Fix a general $S \subset Q$ such that $\sharp(S)=3$ and a general $Y \in L(1,1,0)$. Set $X:=2 L \cup 2 R \cup 2 S \cup Y$. It is sufficient to prove that $h^{1}\left(\mathcal{I}_{X}(5)\right)=0$. Since $X \cap Q$ is a union of a divisor of type (4,0), 32 -points of $Q$ and two points, we have $h^{1}\left(Q, \mathcal{I}_{X \cap Q}(5)\right)=0$. Since $\operatorname{Res}_{Q}(X)=L \cup R \cup Y \cup S$, by the Castelnuovo's sequence it is sufficient to prove that $h^{1}\left(\mathcal{I}_{L \cup R \cup Y \cup S}(3)\right)=0$. We have $h^{1}\left(\mathcal{I}_{L \cup R \cup Y}(3)\right)=0$ by Lemma 1 and hence $h^{0}\left(\mathcal{I}_{L \cup R \cup Y}(3)\right)=4$. Since $S$ is general in $Q$ and $\sharp(S) \leq 4$, it is sufficient to observe that $h^{0}\left(\mathcal{I}_{Y}(1)\right)=0$ (Lemma 3).

(iv) Take $(t, a, \beta)=(0,6,0)$. Take $L, R \in\left|\mathcal{O}_{Q}(1,0)\right|$ with $L \neq R$. Fix a general $S \subset Q$ with $\sharp(S)=4$ and a general $Z \in L(0,2,0)$. Set $X=Z \cup 2 L \cup 2 R \cup$ $2 S$. It is sufficient to prove that $h^{i}\left(\mathcal{I}_{X}(5)\right)=0$. Since $X \cap Q$ is a the union of a divisor of type $(4,0)$ and 3 general 2-points of $Q$, we have $h^{i}\left(Q, \mathcal{I}_{X \cap Q}(5)\right)=0$, $i=0,1$ ([14, Proposition 5.2 and Theorem 7.2]). Hence it is sufficient to prove that $h^{i}\left(\mathcal{I}_{Z \cup L \cup R \cup S}(3)\right)=0, i=0,1$. We first check that $h^{1}\left(\mathcal{I}_{Z \cup L \cup R}(3)\right)=0$. Since any two disjoint lines of $\mathbb{P}^{3}$ are contained in a smooth quadric, $Z \cup L \cup R$ may be considered as a general element of $L(2,2,0)$. Hence $h^{1}\left(\mathcal{I}_{Z \cup L \cup R}(3)\right)=0$ and $h^{0}\left(\mathcal{I}_{Z \cup L \cup R}(3)\right)=4$. Since $S$ is general in $Q, \operatorname{Res}_{Q}(Z \cup L \cup R)=Z$ and $\sharp(S)=4$, to prove that $h^{i}\left(\mathcal{I}_{Z \cup L \cup R \cup S}(3)\right)=0, i=0,1$, it is sufficient to use the obvious observation that $h^{0}\left(\mathcal{I}_{Z}(1)\right)=0$ (Lemma 3$)$.

Lemma 30. We have $h^{0}\left(\mathcal{I}_{X(t, a, 1)}(5)\right)=0$ for all $(a, t) \in \mathbb{N}^{2}$ such that $6 t+4 a+16 \geq 56$.

Proof. Set $\beta^{\prime}:=6 t+4 a-40$. Increasing if necessary $a$ we reduce to the case $0 \leq \beta^{\prime} \leq 3$. Notice that $\beta^{\prime}$ is even. Since all cases with $\beta^{\prime}=0$ are covered by Lemma 29 , it is sufficient to check all cases with $\beta^{\prime}=2$, i.e. the following pairs $(t, a):(7,0),(5,3),(3,6)$, and $(1,9)$.

(i) Assume $(t, a)=(7,0)$. Take a disjoint union $E \in\left|\mathcal{O}_{Q}(3,0)\right|$ of 3 lines and $L \in\left|\mathcal{O}_{Q}(1,0)\right|$ with $L \cap E=\emptyset$. Take a general $Y \in L(4,0,0)$ and set $X:=Y \cup E \cup 2 L$. By the semicontinuity theorem it is sufficient to prove that $h^{0}\left(\mathcal{I}_{X}(5)\right)=0$. Since $X \cap Q$ is the disjoint union of a divisor of type $(5,0)$ and 6 general points, we have $h^{0}\left(Q, \mathcal{I}_{Q \cap X}(5)\right)=0$. Since $\operatorname{Res}_{Q}(X)=Y \cup L$ may be considered as a general union of 5 lines, we have $h^{0}\left(\mathcal{I}_{Y \cup L}(3)\right)=0$ ([13]). Apply the Castelnuovo's sequence.

(ii) Assume $(t, a)=(5,3)$. Fix a general $Y \in L(3,1,0), 3$ distinct lines $L, M, R \in\left|\mathcal{O}_{Q}(1,0)\right|$ and a general $S \subset Q$ such that $\sharp(S)=2$. Set $X:=Y \cup$ $2 S \cup 2 L \cup M \cup R$. By semicontinuity it is sufficient to prove that $h^{0}\left(\mathcal{I}_{X}(5)\right)=0$. 
We have $h^{1}\left(Q, \mathcal{I}_{\{2 S, Q\}}(1,5)\right)=0([14$, Proposition 5.2 and Theorem 7.2]). Since $Y \cap Q$ is a general union of 6 points, we get $h^{i}\left(Q, \mathcal{I}_{X \cap Q}(5)\right)=0, i=0,1$. We have $\operatorname{Res}_{Q}(X)=Y \cup L \cup S$. Since $Y \cup L$, may be considered as a general element of $L(4,1,0)$, we have $h^{i}\left(\mathcal{I}_{Y \cup L}(3)\right)=0, i=0,1$ (Lemma 1). Hence $h^{0}\left(\mathcal{I}_{Y \cup L \cup S}(3)\right)=0$. Use the Castelnuovo's sequence.

(iii) Assume $(t, a)=(3,6)$. Fix a general $Y \in L(3,0,0)$. Take $L \in$ $\left|\mathcal{O}_{Q}(1,0)\right|$ and a general $S \subset Q$ with $\sharp(S)=6$. Set $X:=Y \cup 2 L \cup 2 S$. It is sufficient to prove that $h^{0}\left(\mathcal{I}_{X}(5)\right)=0$. Since $h^{1}\left(Q, \mathcal{I}_{\{2 S, Q\}}(3,5)\right)=0([14$, Proposition 5.2 and Theorem 7.2]) and $Y \cap Q$ is a general union of 6 points of $Q$, we have $h^{i}\left(Q, \mathcal{I}_{X \cap Q}(5)\right)=0, i=0,1$. We have $\operatorname{Res}_{Q}(X)=Y \cup L \cup S$. Since $Y \cup L$ may be considered as a general element of $L(4,0,0)$, we have $h^{0}\left(\mathcal{I}_{Y \cup L}(3)\right)=4$ and $h^{0}\left(\mathcal{I}_{Y \cup L}(1)\right)=0$. Since $S$ is a general union of 6 points of $Q$, Lemma 3 gives $h^{0}\left(\mathcal{I}_{\operatorname{Res}_{Q}(X)}(3)\right)=0$. Apply the Castelnuovo's sequence.

(iv) Assume $(t, a)=(1,9)$. Fix $L, R \in \mathcal{O}_{Q}(1,0) \mid$ such that $L \neq R$, a general $Z \in L(0,3,0)$ and a general $S \subset Q$ such that $\sharp(S)=6$. Set $X:=2 L \cup R \cup$ $Z \cup 2 S$. It is sufficient to prove that $h^{0}\left(\mathcal{I}_{X}(5)\right)=0$. Since $h^{i}\left(\mathcal{I}_{\{2 S, Q\}}(2,5)\right)=0$, $i=0,1$ ([14, Proposition 5.2 and Theorem 7.2]), we have $h^{i}\left(Q, \mathcal{I}_{X \cap Q}(5)\right)=0$, $i=0,1$. We have $\operatorname{Res}_{Q}(X)=L \cup Z \cup S$. Since $h^{1}\left(\mathcal{I}_{Z}(3)\right)=0$ (Lemma 1), we have $h^{0}\left(\mathcal{I}_{Z \cup L}(3)\right)=4$. Obviously $h^{0}\left(\mathcal{I}_{Z}(1)\right)=0$. Since $\operatorname{Res}_{Q}(Z \cup L)=Z$ and $S$ is a general union of 6 points of $Q$, Lemma 3 gives $h^{0}\left(\mathcal{I}_{Z \cup L \cup S}(3)\right)=0$. The Castelnuovo's sequence gives $h^{0}\left(\mathcal{I}_{X}(5)\right)=0$.

Lemma 31. We have $h^{0}\left(\mathcal{I}_{X(t, a, 2)}(5)\right)=0$ for all $(a, t) \in \mathbb{N}^{2}$ such that $6 t+4 a+32 \geq 56$.

Proof. Set $\beta^{\prime}:=6 t+4 a-24$. Notice that $\beta^{\prime}$ is even. Increasing if necessary $a$ we reduce to the case $0 \leq \beta^{\prime} \leq 3$. Since all cases with $\beta^{\prime}=0$ are covered by Lemma 29, we may assume $\beta^{\prime}=2$. Hence either $(t, a)=(3,2)$ or $(t, a)=(1,5)$.

(i) Assume $(t, a)=(3,2)$. Fix lines $L, R \in\left|\mathcal{O}_{Q}(1,0)\right|$, a general $S \subset Q$ with $\sharp(S)=2$ and a general $Y \in L(3,0,0)$. Set $X:=2 L \cup 2 R \cup Y \cup 2 S$. It is sufficient to prove that $h^{0}\left(\mathcal{I}_{X}(5)\right)=0$. Since $h^{1}\left(Q, \mathcal{I}_{\{2 S, Q\}}(1,5)\right)=0([14$, Proposition 5.2 and Theorem 7.2]) and $Y \cap Q$ is a general union of 6 points, we have $h^{i}\left(Q, \mathcal{I}_{X \cap Q}(5)\right)=0, i=0,1$. Since (for a general $Q$ ) $Y \cup L$ is a general union of 4 lines, we have $h^{0}\left(\mathcal{I}_{Y \cup L}(3)\right)=0$. Obviously $h^{0}\left(\mathcal{I}_{Y}(1)\right)=0$. Since $\operatorname{Res}_{Q}(X)=Y \cup L \cup S$ and $S$ is a general union of 6 points of $Q$, Lemma 3 gives $h^{0}\left(\mathcal{I}_{Y \cup L \cup S}(3)\right)=0$. Apply the Castelnuovo's sequence.

(ii) Assume $(t, a)=(1,5)$. Fix lines $L, R \in\left|\mathcal{O}_{Q}(1,0)\right|$, a general $S \subset Q$ with $\sharp(S)=4$ and a general $Y \in L(1,1,0)$. Set $X:=Y \cup 2 S \cup 2 L \cup 2 R$. It is sufficient to prove that $h^{0}\left(\mathcal{I}_{X}(5)\right)=0$. Since $h^{i}\left(\mathcal{I}_{\{2 S, Q\}}(1,5)\right)=0, i=0,1$ ([14, Proposition 5.2 and Theorem 7.2]), we have $h^{0}\left(Q, \mathcal{I}_{X \cap Q}(5)\right)=0$. We 
have $\operatorname{Res}_{Q}(X)=Y \cup L \cup R \cup S$. Since any two disjoint lines are contained in a smooth quadric, $Y \cup L \cup R$ may be considered as a general element of $L(3,1,0)$. Hence $h^{0}\left(\mathcal{I}_{Y}(3)=4\right.$ (Lemma 1$)$. Since $h^{0}\left(\mathcal{I}_{Y}(1)\right)=0$, Lemma 3 gives $h^{0}\left(\mathcal{I}_{Y \cup L \cup R \cup S}(3)\right)=0$. Use the Castelnuovo's sequence.

Lemma 32. We have $h^{0}\left(\mathcal{I}_{X(t, a, 3)}(5)\right)=h^{0}\left(\mathcal{I}_{X(t, a, 0)}(3)\right)$ and $h^{1}\left(\mathcal{I}_{X(t, a, 3)}(5)\right)$ $\geq 2 t$. If either $1 \leq t \leq 4$ and $t+a \leq 4$ or $(t, a)=(2,3)$, then

$$
h^{0}\left(\mathcal{I}_{X(t, a, 3)}(5)\right) \cdot h^{0}\left(\mathcal{I}_{X(t, a, 3)}(5)\right)>0 .
$$

If $5 t+4 a \leq 35$, then $h^{1}\left(\mathcal{I}_{X(t, a, 3)}(x)\right)=0$ for all $x \geq 6$.

Proof. Write $X(t, a, c)=2 L_{1} \sqcup 2 L_{2} \sqcup 2 L_{3} \sqcup B \sqcup A$ with $L_{1}, L_{2}, L_{3}$ skew lines, $B$ general in $L(t, 0,0)$ and $A$ general in $L(0, a, 0)$. Let $Q^{\prime}$ be the unique smooth quadric containing $L_{1} \cup L_{2} \cup L_{3}$, say as an element of $\left|\mathcal{O}_{Q}(3,0)\right|$. Since $\left(2 L_{1} \cup 2 L_{2} \cup 2 L_{3}\right) \cap Q \in\left|\mathcal{O}_{Q}(6,0)\right|$ and $B \cap Q$ is a general union of $2 t$ points of $Q$ and $A \cap Q=\emptyset$, we have $h^{0}\left(Q, \mathcal{I}_{X(t, a, c)}(5)\right)=0$ and $h^{1}\left(Q, \mathcal{I}_{X(t, a, c)}(5)\right)=2 t$. Since $\operatorname{Res}_{Q}(X(t, a, c))=B \cup A$ is a general element of $L(t, a, 0)$ and $h^{2}\left(\mathcal{I}_{A \cup B}(3)\right)=$ $h^{1}\left(\mathcal{O}_{A \cup B}(3)\right)=0$, the Castelnuovo's sequence gives

$$
h^{0}\left(\mathcal{I}_{X(t, a, 3)}(5)\right)=h^{0}\left(\mathcal{I}_{X(t, a, 0)}(3)\right)
$$

and

$$
h^{1}\left(\mathcal{I}_{X(t, a, 3)}(5)\right) \geq 2 t .
$$

The second part follows from Lemma 1, i.e. from [4]. The third part follows from the Castelnuovo's sequence, because $h^{1}\left(\mathcal{I}_{X(t, a, 0)}(x-2)\right)=0$ (Lemma $1)$.

Lemma 33. We have

$$
\begin{gathered}
h^{0}\left(\mathcal{I}_{X(0,0,4)}(5)\right)=0, \\
h^{1}\left(\mathcal{I}_{X(0,0,4)}(6)\right)=h^{0}\left(\mathcal{I}_{X(0,0,4)}(6)\right)=2
\end{gathered}
$$

and

$$
h^{1}\left(\mathcal{I}_{X(0,0,4)}(x)\right)=0
$$

for all $x \geq 7$.

Proof. Set $D:=X(0,0,4)_{\text {red }}$ and write $D=L \sqcup B$ with $B \in L(3,0,0)$. Let $Q^{\prime}$ be the only smooth quadric containing $B$, say as lines of type $(1,0)$. The scheme $X(0,0,4) \cap Q^{\prime}$ is a disjoint union of a divisor of type $(6,0)$ and two general 2-points of $Q$. Hence $h^{0}\left(Q^{\prime}, \mathcal{I}_{Q^{\prime} \cap X(0,0,4)}(5)\right)=0, h^{0}\left(\mathcal{I}_{Q^{\prime} \cap X(0,0,4)}(6)\right)=2$, 
$h^{1}\left(Q^{\prime}, \mathcal{I}_{Q^{\prime} \cap X(0,0,4)}(6)\right)=2$ and $h^{1}\left(Q^{\prime}, \mathcal{I}_{Q^{\prime} \cap X(0,0,4)}(x)\right)=0$ for all $x \geq 7$. The scheme $\operatorname{Res}_{Q^{\prime}}(X(0,0,4))=2 L \sqcup B$ is a general element of $L(3,0,1)$. We have $h^{0}\left(\mathcal{I}_{2 L \cup B}(3)\right)=0$ (Lemma 9$)$ and $h^{1}\left(\mathcal{I}_{2 L \cup B}(y)\right)=0$ for all $y \geq 4$ (Remark 3 and Lemma 13). We have $h^{2}\left(\mathcal{I}_{2 L \cup B}(4)\right)=0$. Use several times the Castelnuovo's sequence.

Lemma 34. We have

$$
h^{1}\left(\mathcal{I}_{X(t, a, 1)}(6)\right)=0
$$

for all $(t, a) \in \mathbb{N}^{2}$ such that $7 t+4 a \leq 65$ and

$$
h^{0}\left(\mathcal{I}_{X(t, a, 1)}(6)\right)=0
$$

for all $(t, a) \in \mathbb{N}^{2}$ such that $7 t+4 a \geq 65$ (for the latter part we assume that either $t \leq 4$ or the characteristic is zero).

Proof. In all cases by semicontinuity it is sufficient to find $X \in L(t, a, 1)$ such that $h^{0}\left(\mathcal{I}_{X}(6)\right) \cdot h^{1}\left(\mathcal{I}_{X}(6)\right)=0$.

(a) In this step we prove the first statement. Set $\beta:=65-7 t-4 b$. Increasing if necessary $b$ we reduce to the case $0 \leq \beta \leq 3$. We have the following triples $(t, a, \beta):(9,0,2),(8,2,1),(7,4,0),(6,5,3),(5,7,2),(4,9,1)$, $(3,11,0),(2,12,3),(1,14,2),(0,16,1)$.

(a1) Assume $(t, a)=(9,0)$. Take $L \in\left|\mathcal{O}_{Q}(1,0)\right|$ and $E \in\left|\mathcal{O}_{Q}(3,0)\right|$ with 3 connected components and $E \cap L=\emptyset$. Fix a general $Y \in L(6,0,0)$ and take $X:=Y \cup E \cup 2 L$. We have $\operatorname{Res}_{Q}(X)=Y \cup L$. Since $Y \cup L$ may be considered as a general union of 7 lines, we have $h^{i}\left(\mathcal{I}_{Y \cup L}(4)\right)=0, i=0,1$. Therefore by the Castelnuovo's sequence it is sufficient to prove that $h^{1}\left(Q, \mathcal{I}_{X \cap Q}(6)\right)=0$. This is true, because $X \cap Q$ is the union of a divisor of type $(5,0)$ and 12 general points.

(a2) Assume $(t, a)=(8,2)$. Take $L \in\left|\mathcal{O}_{Q}(1,0)\right|$ and $E \in\left|\mathcal{O}_{Q}(3,0)\right|$ with 3 connected components and $E \cap L=\emptyset$. Fix a general $O \in Q$ and a general $Y \in L(5,1,0)$. Set $X:=Y \cup E \cup 2 O \cup 2 L$. We have $\operatorname{Res}_{Q}(X)=Y \cup L \cup\{O\}$. Lemma 1 gives $h^{1}\left(\mathcal{I}_{Y \cup L}(4)\right)=0$. Obviously $h^{0}\left(\mathcal{I}_{Y}(2)\right)=0$. Since $O$ is general in $Q$, we get $h^{i}\left(\mathcal{I}_{\operatorname{Res}_{Q}(X)}(4)\right)=0, i=0,1$ (Lemma 3). By the Castelnuovo's sequence it is sufficient to prove that $h^{1}\left(Q, \mathcal{I}_{X \cap Q}(6)\right)=0$. This is true, because $X \cap Q$ is a general union of a divisor of type $(5,0)$, a general 2-point of $Q$ and 10 general points of $Q$.

(a3) Assume $(t, a)=(7,4)$. Take $L \in\left|\mathcal{O}_{Q}(1,0)\right|$ and $E \in\left|\mathcal{O}_{Q}(3,0)\right|$ with 3 connected components and $E \cap L=\emptyset$. Fix a general $S \subset Q$ such that $\sharp(S)=2$. Fix a general $Y \in L(4,2,0)$ and set $X:=Y \cup 2 L \cup E \cup 2 S$. Since 
$X \cap Q$ is a general union of a divisor of type $(5,0)$, two general 2-point of $Q$ and 8 general points, we have $h^{i}\left(Q, \mathcal{I}_{X \cap Q}(6)\right)=0$ ([14, Proposition 5.2 and Theorem 7.2]). By the Castelnuovo's sequence it is sufficient to prove that $h^{i}\left(\mathcal{I}_{\operatorname{Res}_{Q}(X)}(4)\right)=0, i=0,1$. We have $\operatorname{Res}_{Q}(X)=Y \cup L \cup S$. Since $Y \cup L$ may be considered as a general element of $L(5,2,0)$, we have $h^{1}\left(\mathcal{I}_{Y \cup L}(4)\right)=0$. Obviously $h^{0}\left(\mathcal{I}_{Y}(2)\right)=0$. Use Lemma 3 .

(a4) Assume $0 \leq t \leq 6$. Set $\alpha_{t}:=3$ if $3 \leq t \leq 6, \alpha_{2}=\alpha_{1}=1$ and $\alpha_{0}=0$. If $t \neq 2$, then set $e_{t}:=\left\lfloor\left(7\left(5-\alpha_{t}\right)-2\left(t-\alpha_{t}\right)\right) / 3\right\rfloor$ and $f_{t}:=$ $7\left(5-\alpha_{t}\right)-3 e_{t}-2\left(t-\alpha_{t}\right)$. Set $e_{2}:=6$ and $f_{2}=0$. We have $\left(e_{6}, f_{6}\right)=(2,2)$, $\left(e_{5}, f_{5}\right)=(3,1),\left(e_{4}, f_{4}\right)=(4,0),\left(e_{3}, f_{3}\right)=(4,2),\left(e_{1}, f_{1}\right)=(9,1) . \quad$ In all cases we have $a \geq e_{t}+f_{t}$. Fix $L \in\left|\mathcal{O}_{Q}(1,0)\right|$ and $E \in\left|\mathcal{O}_{Q}\left(\alpha_{t}, 0\right)\right|$ with $E$ with $\alpha_{t}$ connected components and $E \cap L=\emptyset$. Fix a general $S \cup S^{\prime} \subset Q$ such that $\sharp(S)=\alpha_{t}, \sharp\left(S^{\prime}\right)=f_{t}$ and $S \cap S^{\prime}=\emptyset$. Fix a general $Y \in L(t-$ $\left.\alpha_{t}, a-e_{t}-f_{t}, 0\right)$. Set $X_{1}:=2 L \cup Y \cup 2 S$. By the differential Horace lemma for 2-points ([1], [11, Lemma 5]), to prove that a general union $X$ of $X_{1}$ and $f_{t}$ 2-points (and hence to prove the lemma in this case) it is sufficient to prove that $h^{1}\left(Q, \mathcal{I}_{\left(X_{1} \cup Q\right) \cup S^{\prime}}(6)\right)=0$ and that $h^{1}\left(\mathcal{I}_{Y \cup L \cup S \cup\left\{2 S^{\prime}, Q\right\}}(4)\right)=0$. We first check that $h^{1}\left(\mathcal{I}_{Y \cup L \cup\left\{2 S^{\prime}, Q\right\}}(4)\right)=0$. By the last part of Remark 3 it is sufficient to prove that $h^{1}\left(\mathcal{I}_{Y \cup L \cup 2 S^{\prime}}(4)\right)=0$. In all cases we have $h^{0}\left(\mathcal{O}_{Y \cup L \cup 2 S^{\prime}}(4)\right) \leq 35$. Hence Lemma 1 gives $h^{1}\left(\mathcal{I}_{Y \cup L \cup 2 S^{\prime}}(4)\right)=0$. In all cases we have $h^{0}\left(\mathcal{O}_{\left(X_{1} \cap Q\right) \cup S^{\prime}}(6)\right) \leq 49$. In all cases we have $h^{1}\left(Q, \mathcal{I}_{\left(X_{1} \cap Q\right) \cup S^{\prime}}(6)\right)=0$ by [14, Proposition 5.2 and Theorem 7.2]; we chose the values of $\alpha_{2}, e_{2}$ and $f_{2}$ to avoid the case $(2,6)$ with 72 -points of $Q$, which in the list of exceptional cases.

(b) Now we prove the second statement. Set $\beta^{\prime}:=7 t+4 b-65$. Decreasing if necessary $a$ and, if $a=0$, decreasing also $t$ we reduce to the cases $0 \leq \beta^{\prime} \leq 3$ and the cases $a=0$ and $0 \leq \beta^{\prime} \leq 7$. Since the first part covered all cases with $\beta^{\prime}=0$, we may also assume $\beta^{\prime}>0$. Hence we need to check the following triples $\left(t, a, \beta^{\prime}\right):(10,0,5),(9,1,2),(8,3,3),(6,6,1),(5,8,2)$, $(4,10,3),(2,13,1),(1,14,2),(0,17,3)$. However, since a 2 -point contains a point, the case $\left(t, a, \beta^{\prime}\right)=(0,17,3)$ follows from the case $(t, a, \beta)=(0,16,1)$, the case $\left(t, a, \beta^{\prime}\right)=(8,3,3)$ follows from the case $(t, a, \beta)=(8,2,2)$ and the case $\left(t, a, \beta^{\prime}\right)=(4,10,3)$ follows from the case $(t, a, \beta)=(4,9,1)$ (cases proved in step (a)). A 2-point contains a tangent vector. Hence in characteristic zero all cases with $\beta^{\prime}=2$ are true by [12] or [8, Lemma 1.8]; to cover Theorem 1 in arbitrary characteristic we check in arbitrary characteristic the case $(t, a)=(1,14)$. Any two points of $\mathbb{P}^{3}$ are contained in a line. Hence the case $\left(t, a, \beta^{\prime}\right)=(10,0,5)$ of part (b) follows from the case $(t, a, \beta)=(9,0,2)$ of part (a).

(b1) Take $\left(t, a, \beta^{\prime}\right)=(6,6,1)$. Fix $L \in \mathcal{O}_{Q}(1,0)$, a general $E \in\left|\mathcal{O}_{Q}(2,0)\right|$, 
a general $S \subset Q$ with $\sharp(S)=4$, a general $O \in Q$ and a general $Y \in L(4,1,0)$. Set $X^{\prime}:=Y \cup E \cup 2 L \cup 2 S$. We have $\operatorname{Res}_{Q}\left(X^{\prime}\right)=Y \cup L \cup S$. Moreover we have $h^{i}\left(Q, \mathcal{I}_{\left(Q \cap X^{\prime}\right) \cup\{O\}}(6)\right)=0$, because $\{O\} \cup(Y \cap Q)$ is a general union of 9 points of $Q$ and $h^{0}\left(Q, \mathcal{I}_{\{2 S, Q\}}(2,6)\right)=9$. By the differential Horace lemma for 2-points ([1], [11, Lemma 5]) to prove that a general union of $X^{\prime}$ and a 2-point (and hence to prove the lemma in this case), it is sufficient to prove that $h^{0}\left(\mathcal{I}_{Y \cup L \cup S \cup\{2 O, Q\}}(4)\right)=0$. Lemma 1 gives $h^{1}\left(\mathcal{I}_{Y \cup L \cup 2 O}(4)\right)=0$ and hence $h^{1}\left(\mathcal{I}_{Y \cup L \cup\{2 O, Q\}}(4)\right)=0$ (last part of Remark 3), i.e. $h^{0}\left(\mathcal{I}_{Y \cup L \cup\{2 O, Q\}}(4)\right)=3$. Obviously $h^{0}\left(\mathcal{I}_{Y}(2)\right)=0$. Since $S$ is general, Lemma 3 gives

$$
h^{0}\left(\mathcal{I}_{Y \cup L \cup S \cup\{2 O, Q\}}(4)\right)=0 .
$$

(b2) Assume $\left(t, a, \beta^{\prime}\right)=(2,13,1)$. Fix $L, R \in\left|\mathcal{O}_{Q}(1,0)\right|$ with $L \neq R$. Fix a general $S \subset Q$ such that $\sharp(S)=9$ and a general $Y \in L(1,4,0)$. Set $X:=Y \cup 2 L \cup R \cup M \cup 2 S$. We have $\operatorname{Res}_{Q}(X)=Y \cup L \cup S$. Lemma 1 gives $h^{1}\left(\mathcal{I}_{Y \cup L}(4)\right)=0$ and $h^{0}\left(\mathcal{I}_{Y \cup L}(4)\right)=9$. Obviously $h^{0}\left(\mathcal{I}_{Y}(2)\right)=0$. Since $S$ is general in $Q$, part (i) of Lemma 3 gives $h^{0}\left(\mathcal{I}_{\operatorname{Res}_{Q}(X)}(4)\right)=0$. We have $h^{i}\left(\mathcal{I}_{X \cap Q}(6)\right)=0, i=0,1$, because $h^{1}\left(\mathcal{I}_{\{2 S, Q\}}(3,6)\right)=0$ ([14, Proposition 5.2 and Theorem 7.2]), i.e. $h^{0}\left(\mathcal{I}_{\{2 S, Q\}}(3,6)\right)=1$, and $Y \cap Q$ is a general union of two points of $Q$.

(b3) Assume $\left(t, a, \beta^{\prime}\right)=(1,14,2)$. Fix $L, R \in\left|\mathcal{O}_{Q}(1,0)\right|$ with $L \neq R$. Fix a general $S \subset Q$ such that $\sharp(S)=10$ and a general $Y \in L(0,4,0)$. Set $X:=$ $Y \cup 2 L \cup 2 S$. We have $h^{0}\left(Q, \mathcal{O}_{Q \cap X}(6)\right)=0$, because $h^{0}\left(Q, \mathcal{I}_{\{2 S, Q\}}(3,6)\right)=0([14$, Proposition 5.2 and Theorem 7.2]). By the Castelnuovo's sequence it is sufficient to prove that $h^{i}\left(\mathcal{I}_{\operatorname{Res}_{Q}(X)}(4)\right)=0, i=0,1$. We have $\operatorname{Res}_{Q}(X)=Y \cup L \cup S$. Lemma 1 gives $h^{1}\left(\mathcal{I}_{Y \cup L}(4)\right)=0$ and hence $h^{0}\left(\mathcal{I}_{Y \cup L}(4)\right)=10$. Since the singular locus of a quadric is a proper linear subspace, we have $h^{0}\left(\mathcal{I}_{Y}(2)\right)=0$. Since the finite set $S$ is general in $Q$ and $\sharp(S)=10$, Lemma 3 gives $h^{i}\left(\mathcal{I}_{Y \cup L \cup S}(4)\right)=0$, $i=0,1$.

Lemma 35. We have $h^{1}\left(\mathcal{I}_{X(t, a, c)}(6)\right) \geq 2 c-6$.

Proof. We may assume $c \geq 4$. Write $X(t, a, c)=A \sqcup L_{1} \sqcup \cdots \sqcup L_{c}$ with $A \in L(t, a, 0)$ and $L_{1}, \ldots, L_{c}$ lines. Let $Q$ be the smooth quadric containing $L_{1} \cup L_{2} \cup L_{3}$. Call $\left|\mathcal{O}_{Q}(1,0)\right|$ the ruling of $Q$ containing $L_{1}$. The generality of $X(t, a, c)$ gives that $X(t, a, c) \cap Q$ is a general union of a divisor of type $(6,0)$, $2 c-62$-points and $2 t$ points. Hence $h^{1}\left(Q, \mathcal{I}_{X(t, 0, c) \cap Q}(6)\right) \geq 2 c-6$. Use the Castelnuovo's sequence and that $h^{2}\left(\mathcal{I}_{X(t+3, a, c-3)}(4)\right)=0$.

Remark 5. By Lemmas 13 and 35 we have $h^{0}\left(\mathcal{I}_{X(t, a, c)}(6)\right) \cdot h^{1}\left(\mathcal{I}_{X(t, a, c)}(6)\right)>$ 0 if either $(t, a, c)=(0,0,4)$ or $(t, a, c)=(0,1,4)$. 


\section{Proof of Theorem 1}

Consider the following assertion $H_{k}, k \geq 5$ :

Assertion $H_{k}$ : Fix nonnegative integers $t, c, a$ such that $t \leq\lfloor(k+$ $\left.2)^{2} / 64\right\rfloor-\left\lfloor k^{2} / 64\right\rfloor, 3 t+c \leq\left\lfloor k^{2} / 64\right\rfloor$ and $4 a+t(k+1)+(3 k+5) c \leq\left(\begin{array}{c}k+3 \\ 3\end{array}\right)$. Then there exists $W \in L(t, a, c)$ such that $h^{1}\left(\mathcal{I}_{W}(k)\right)=0$, the $t$ lines of $W$ are contained in a smooth quadric $Q$ and two of the 2-points of $W$ have support contained in the same quadric $Q$.

Proof of Theorem 1 and of Assertion $H_{k}$ : Recall that $k \geq 5$. Fix any $A \in$ $L(t, a, c)$ and assume $h^{1}\left(\mathcal{I}_{A}(k)\right)=0$. Since $k \geq 2$, we have $h^{2}\left(\mathcal{I}_{A}(k-1)\right)=$ $h^{1}\left(\mathcal{O}_{A}(k-1)\right)=0$. Hence Castelnuovo-Mumford's lemma gives $h^{1}\left(\mathcal{I}_{A}(x)\right)=0$ for all $x>k$. See step (iii) for the case $6 \leq k \leq 19$. Before step (iii) we assume $k \geq 20$ and that $H_{k^{\prime}}$ and Theorem 1 are true for all integers $k^{\prime}<k$. In step (i) we prove $H_{k}$ and part (i) of Theorem 1, while in step (ii) we consider part (ii) of Theorem 1.

(i) In this step we assume $t(k+1)+c(3 k+1)+4 a \leq\left(\begin{array}{c}k+3 \\ 3\end{array}\right)$. Set $\beta:=$ $\left(\begin{array}{c}k+3 \\ 3\end{array}\right)-(k+1) t-4 a-(3 k+1) c$. Adding if necessary several 2-points we see that it is sufficient to do the cases with $0 \leq \beta \leq 3$. Since Theorem 1 is true if $k^{\prime}<k$, sometimes we assume $3 t+c>\left\lfloor(k-1)^{2} / 64\right\rfloor$ (not always, because to get $H_{k}$ if $3 t+c \leq 3 t+c>\left\lfloor(k-1)^{2} / 64\right\rfloor$ we need an additional argument).

(i1) Assume $c \leq\left\lfloor(k-2)^{2} / 64\right\rfloor$. Set $t_{1}:=\left\lfloor\left(\left\lfloor(k-2)^{2} / 64\right\rfloor-c\right) / 3\right\rfloor$, i.e. let $t_{1}$ be the maximal integer such that $3 t_{1}+c \leq\left\lfloor(k-2)^{2} / 64\right\rfloor$. Our assumption on $c$ implies $t_{1} \geq 0$ and $0 \leq\left\lfloor(k-2)^{2} / 64\right\rfloor-c-3 t_{1} \leq 2$. Since $3 t+c>\left\lfloor(k-1)^{2} / 64\right\rfloor$, we have $t>t_{1}$ (for $H_{k}$ see step (i1.1)).

Claim 1: We have $t-t_{1} \leq k-3$.

Proof of Claim 1: Assume $t-t_{1} \geq k-2$. Since $3 t+c \leq\left\lfloor k^{2} / 64\right\rfloor$ and $3 t_{1}+c \geq\left\lfloor(k-2)^{2} / 64\right\rfloor-2$, we get $3(k-2)+2 \geq\left\lfloor k^{2} / 64\right\rfloor-\left\lfloor(k-2)^{2} / 64\right\rfloor$, which is false for all $k \geq 5$.

Let $U \subset Q$ be a general union of $t-t_{1}$ lines of type $(1,0)$. Set $u:=$ $\left\lfloor\left((k+1)\left(k+1+t_{1}-t\right)-2 t_{1}-6 c\right) / 3\right\rfloor$ and $v:=(k+1)\left(k+1+t_{1}-t\right)-2 t_{1}-6 c$. We have $0 \leq v \leq 2$ and $3 u+v+2 t_{1}+6 c=(k+1)^{2}$.

Claim 2: We have $u \geq 2$.

Proof of Claim 2: Assume $u \leq 1$. Since $v \leq 2$, we get $5+2 t_{1}+6 c \geq$ $(k+1)^{2}$. Since $t_{1} \leq t$ and $3 t+c \leq k^{2} / 64$, we get a contradiction.

Fix a general $S \cup S^{\prime} \subset Q$ such that $\sharp(S)=u, \sharp\left(S^{\prime}\right)=v$ and $S \cap S^{\prime}=\emptyset$ (we are using that $u \geq 0$ and this inequality is true by Claim 2).

Claim 3: We have $a \geq u+v$. 
Proof of Claim 3: Write $u_{k}$ and $v_{k}$ if we need to make explicit the dependence on $k$. Since $3 u+v+2 t_{1}+6 c=(k+1)^{2}$ and $3 t_{1}+c \geq(k-2)^{2} / 64-3$, we have $3 u+v \leq(k+1)^{2}-(k-2)^{2} / 96+2$. We get $3 u_{10}+v_{10} \leq 122,3 u_{11}+v_{11} \leq 145$, $3 u_{12}+v_{12} \leq 170$. Since $\beta \leq 3$ and $3 t+c \leq k^{2} / 64$, we have $4 a+(3 k+1) k^{2} / 64 \geq$ $\left(\begin{array}{c}k+3 \\ 3\end{array}\right)-3$; if $a \leq u+v-1$, then $4 u+4 v+(3 k+1) k^{2} / 64 \geq\left(\begin{array}{c}k+3 \\ 3\end{array}\right)+1$. We get $4 u_{10}+4 v_{10} \geq 238,4 u_{11}+4 v_{11} \geq 301,4 u_{12}+4 v_{12} \geq 373$. Since $v \leq 2$, we get a contradiction if $k=10,11,12$. Since $v \leq 2$ the study of the function $\psi(k)=\left(\left(\begin{array}{c}k+3 \\ 3\end{array}\right)+1-(3 k+1) k^{2} / 64-8\right) / 4-\left((k+1)^{2}-(k-2)^{2} / 96+2\right) / 3$ gives a contradiction for all $k \geq 13$, concluding the proof of Claim 3 .

Fix a general $Y \in L\left(t_{1}, a-u-v, c\right)$ (it is defined, because $a-u-v \geq 0$ by Claim 3).

By Claim 1 we have $k-t+t_{1} \geq 3$. Since $Y \cap Q$ is a general union of $2 t_{1}+6 c$ 2-points of $Q, S \cup S^{\prime}$ is general in $Q$ and $3 u+v=(k+1)\left(k+1+t_{1}-t\right)$, we get $h^{i}\left(Q, \mathcal{I}_{U \cup(Y \cap Q) \cup S^{\prime} \cup\{2 S ; Q\}}(k)\right)=0$. By the differential Horace lemma for double points $\left([1]\right.$, [11, Lemma 5]) to prove that $h^{1}\left(\mathcal{I}_{M}(k)\right)=0$ for a general union $M$ of $Y \cup U \cup 2 S$ and $v$ general 2-points it is sufficient to prove that $h^{1}\left(\mathcal{I}_{Y \cup S \cup\left\{2 S^{\prime}, Q\right\}}(k-2)\right)=0$.

Claim 4: $h^{1}\left(\mathcal{I}_{Y \cup\left\{2 S^{\prime}, Q\right\}}(k-2)\right)=0$.

Proof of Claim 4: It is sufficient to prove that $h^{1}\left(\mathcal{I}_{Y \cup 2 S^{\prime}}(k-2)\right)=0$. Since $v \leq 2$ and any two points of $\mathbb{P}^{3}$ are contained in a smooth quadric, $Y \cup 2 S$ has the Hilbert function of $X\left(t_{1}, a-u, c\right)$. We have $h^{0}\left(\mathcal{O}_{X\left(t_{1}, a-u, c\right)}(k-2)\right)=$ $\left(\begin{array}{c}k+1 \\ 3\end{array}\right)-\beta-u+v$. Claim 2 gives $h^{0}\left(\mathcal{O}_{X\left(t_{1}, a-u, c\right)}(k-2)\right) \leq\left(\begin{array}{c}k+1 \\ 3\end{array}\right)$. The inductive assumption gives $h^{1}\left(\mathcal{I}_{Y \cup 2 S^{\prime}}(k-2)\right)=0$, proving Claim 4.

By Claim 4 we have $h^{0}\left(\mathcal{I}_{Y \cup\left\{2 S^{\prime}, Q\right\}}(k-2)\right)=\beta+u$. By Lemma 3 and Claim 4 to prove that $h^{1}\left(\mathcal{I}_{Y \cup S \cup\left\{2 S^{\prime}, Q\right\}}(k-2)\right)=0$ it is sufficient to prove that $h^{0}\left(\mathcal{I}_{Y}(k-4)\right) \leq \beta$. We have

$$
(k-1) t_{1}+(3 k-5) c+4(a-u-v)+u+3 v=\left(\begin{array}{c}
k+1 \\
3
\end{array}\right)-\beta
$$

Recall that $(k-2)^{2} / 64-2 \leq 3 t_{1}+c \leq(k-2)^{2} / 64$. Hence there are integers $t^{\prime}, c^{\prime}$ such that $0 \leq t^{\prime} \leq t_{1}, 0 \leq c^{\prime} \leq c$ and $(k-4)^{2} / 64-3<3 t^{\prime}+c^{\prime} \leq(k-4)^{2} / 64$. Take $M \in L\left(t^{\prime}, a-u-v, c^{\prime}\right)$ such that $M \subseteq Y$. Since $k \geq 9$, the inductive assumption gives that either $h^{0}\left(\mathcal{I}_{M}(k-4)\right)=0$ or $h^{1}\left(\mathcal{I}_{M}(k-4)\right)=0$. In the former case we get $h^{0}\left(\mathcal{I}_{M}(k-4)\right)=0$, concluding the proof in this case. Assume $h^{1}\left(\mathcal{I}_{M}(k-4)\right)=0$. We have $h^{0}\left(\mathcal{I}_{M}(k-4)\right)=\left(\begin{array}{c}k-1 \\ 3\end{array}\right)-4(a-u-v)-$ $(k-3) t^{\prime}-(3 k-11) c^{\prime}$. Since $h^{0}\left(\mathcal{I}_{Y}(k-4)\right) \leq h^{0}\left(\mathcal{I}_{M}(k-4)\right)$, from $(3)$ we get that it is sufficient to prove that

$$
(k-1)\left(t-t^{\prime}\right)+(3 k-5)\left(c-c^{\prime}\right)+2 t^{\prime}+6 c^{\prime} \leq(k-1)^{2}
$$


We have $3\left(t_{1}-t^{\prime}\right)+c-c^{\prime} \leq(k-2)^{2} / 64-(k-4)^{2} / 64+3=(k+36) / 16$. We have $2 t^{\prime}+6 c^{\prime} \leq 6(k-4)^{2} / 64$. Hence it is sufficient to have $(3 k-5)(k+36) / 16+$ $3(k-4)^{2} / 32 \leq(k-1)^{2}$. This inequality is true for all $k \geq 9$.

(i1.1) Now we consider $H_{k}$ in the set-up of step (i1). Hence $t \leq\lfloor(k+$ $\left.2)^{2} / 64\right\rfloor-\left\lfloor k^{2} / 64\right\rfloor \leq(k+20) / 16$. We take $Q$ as the smooth quadric. Let $V \subset Q$ be a general union of $t$ lines of type $(1,0)$. Set $u^{\prime}:=\lfloor((k+1)(k+1-t)-6 c) / 3\rfloor$ and $v^{\prime}:=(k+1)(k+1-t)-6 c-3 u^{\prime}$. We have $0 \leq v^{\prime} \leq 2$. Since $t \leq$ $(k+20) / 16$ and $k \geq 20$, the analogous of Claim 1 holds true in this case. Since $t \leq(k+20) / 16$, we have $u^{\prime} \geq 2$, i.e. the analogous of Claim 2 is true in this case. Fix a general $S_{1} \cup S_{1}^{\prime} \subset Q$ such that $\sharp\left(S_{1}\right)=u^{\prime}, \sharp\left(S_{1}^{\prime}\right)=v^{\prime}$ and $S_{1} \cap S_{1}^{\prime}=\emptyset$.

Claim 5: We have $a \geq u^{\prime}+v^{\prime}$.

Proof of Claim 5: We have

$$
(3 k-5) c+4\left(a-u^{\prime}-v^{\prime}\right)+u^{\prime}+3 v^{\prime}=\left(\begin{array}{c}
k+1 \\
3
\end{array}\right)-\beta
$$

and $3 u^{\prime}+v^{\prime}+6 c=(k+1)(k+1-t)$ and hence $3 u^{\prime}+v^{\prime} \leq(k+1)^{2}$. Since $u \geq v^{\prime}$, we have $u^{\prime}+3 v^{\prime} \leq 3 u^{\prime}+v^{\prime}$ and hence $u^{\prime}+3 v^{\prime} \leq(k+1)^{2}$. Since $c \leq(k-2)^{2} / 64$ and $\beta \leq 3$, (5) gives $a-u^{\prime}-v^{\prime} \geq 0$ for all $k \geq 10$.

Fix a general $Y^{\prime} \in L\left(0, a-u^{\prime}-v^{\prime}, c\right)$. Since $u^{\prime} \geq 2$ to prove $H_{k}$ for the integers $t, c$ it is sufficient to prove that $h^{1}\left(\mathcal{I}_{W}(k)\right)=0$ for a general union $W$ of $Y \cup V \cup 2 S_{1}$ and $v^{\prime}$ 2-points. Since $k+1-t \leq k-3$, [14, Proposition 5.2 and Theorem 7.2] gives $h^{i}\left(Q, \mathcal{I}_{(Y \cap Q) \cup\left\{2 S_{1}, Q\right\} \cup S_{1}^{\prime}}(k, k-t)\right)=0, i=0,1$. By the differential Horace lemma for double points ([1], [11, Lemma 5]) to prove that $h^{1}\left(\mathcal{I}_{W}(k)\right)=0$ it is sufficient to prove that $h^{1}\left(\mathcal{I}_{Y^{\prime} \cup S_{1} \cup\left\{2 S_{1}^{\prime}, Q\right\}}(k-2)\right)=0$. We first check that $h^{1}\left(\mathcal{I}_{Y^{\prime} \cup S^{\prime} \cup\left\{2 S_{1}^{\prime}, Q\right\}}(k-2)\right)=0$. It is sufficient to check that $h^{1}\left(\mathcal{I}_{Y^{\prime} \cup 2 S_{1}^{\prime}}(k-2)\right)=0$. Since any two points of $\mathbb{P}^{3}$ are contained in a smooth quadric, the schemes $X\left(0, a-u^{\prime}, c\right)$ and $Y^{\prime} \cup 2 S_{1}^{\prime}$ have the same Hilbert function. By (5) we have $h^{0}\left(\mathcal{I}_{Y^{\prime} \cup 2 S_{1}^{\prime}}(k-2)\right)=\left(\begin{array}{c}k+1 \\ 3\end{array}\right)-\beta+v^{\prime}-u^{\prime}$. Since $u^{\prime} \geq v^{\prime}$ and $c \leq\left\lfloor(k-2)^{2} / 64\right\rfloor$, the inductive assumption gives $h^{1}\left(\mathcal{I}_{Y^{\prime} \cup 2 S_{1}^{\prime}}(k-2)\right)=0$ and hence $h^{1}\left(\mathcal{I}_{Y^{\prime} \cup\left\{2 S_{1}^{\prime}, Q\right\}}(k-2)\right)=0$, i.e. $h^{0}\left(\mathcal{I}_{Y^{\prime} \cup\left\{2 S_{1}^{\prime}, Q\right\}}(k-2)\right)=\beta+u^{\prime}$. By Lemma 3 it is sufficient to prove that $h^{0}\left(\mathcal{I}_{Y^{\prime}}(k-4)\right) \leq \beta$.

Assume for the moment $c \geq\left\lfloor(k-4)^{2} / 64\right\rfloor$. Fix $M^{\prime} \in L\left(0, a-u^{\prime}-v^{\prime},\lfloor(k-\right.$ $\left.\left.4)^{2} / 64\right\rfloor\right)$ such that $M^{\prime} \subseteq Y^{\prime}$. By the inductive assumption either $h^{0}\left(\mathcal{I}_{M^{\prime}}(k-\right.$ $4))=0\left(\right.$ and hence $\left.h^{0}\left(\mathcal{I}_{Y^{\prime}}(k-4)\right)=0\right)$ or $h^{1}\left(\mathcal{I}_{M^{\prime}}(k-4)\right)=0$, i.e. $h^{0}\left(\mathcal{I}_{M^{\prime}}(k-\right.$ 4) $)=\left(\begin{array}{c}k-1 \\ 3\end{array}\right)-(3 k-11) c-4\left(a^{\prime}-u^{\prime}-v^{\prime}\right)$. Since $h^{0}\left(\mathcal{I}_{M^{\prime}}(k-4)\right) \geq h^{0}\left(\mathcal{I}_{Y^{\prime}}(k-4)\right)$, in order to obtain a contradiction we may assume $\left(\begin{array}{c}k-1 \\ 3\end{array}\right)-(3 k-11) c-4\left(a^{\prime}-u^{\prime}-v^{\prime}\right) \geq$ $\beta+1$. From (5) we get $6 c+u^{\prime}+3 v^{\prime} \geq k^{2}-2 k$. Since $6 c+t(k+1)+3 u^{\prime}+v^{\prime}=$ $(k+1)^{2}$ and $u^{\prime} \geq v^{\prime}$, we get $t<4$, i.e. $t \leq 3$. First assume $t=3$. We get 
$6 c+3 u^{\prime}+v^{\prime}=k^{2}-k+2$, while $6 c+u^{\prime}+3 v^{\prime} \geq k^{2}-2 k$. Since $v^{\prime} \leq 2$, to get a contradiction it is sufficient to have $2 u^{\prime} \geq k+1$. Assume $2 u^{\prime} \leq k$. Since $6 c \leq 3(k-2)^{2} / 32$ and $6 c+3 u^{\prime}+v^{\prime}=k^{2}-k+2$, we get a contradiction. Now assume $t \leq 2$. Since the union of two disjoint lines and any two points is contained in a smooth quadric, in this case $H_{k}$ for the given $(t, a, c)$ follows from part (i) of Theorem 1 for the same triple $(t, a, c)$.

Now assume $c<\left\lfloor(k-4)^{2} / 64\right\rfloor$. By the inductive assumption either $h^{0}\left(\mathcal{I}_{Y^{\prime}}(k-\right.$ $4))=0$ or $h^{1}\left(\mathcal{I}_{Y^{\prime}}(k-4)\right)$. We may assume that the latter occurs and that $h^{0}\left(\mathcal{I}_{Y^{\prime}}(k-4)\right) \geq \beta+1$, i.e. $4\left(a-u^{\prime}-v^{\prime}\right)+(3 k-11) c \leq\left(\begin{array}{c}k-1 \\ 3\end{array}\right)-\beta-1$. Therefore (5) gives $6 c+u^{\prime}+3 v^{\prime} \geq(k-1)^{2}-1$. Since $6 c+3 u^{\prime}+v^{\prime}+t(k+1)=(k+1)^{2}$ and $u^{\prime} \geq v^{\prime}$, we get $t \leq 3$. Since $c<\left\lfloor(k-4)^{2} / 64\right\rfloor$ and $3 t+c>\left\lfloor(k-1)^{2} / 64\right\rfloor$, we get a contradiction.

(i2) Assume $c>\left\lfloor(k-2)^{2} / 64\right\rfloor$. Set $m:=c-\left\lfloor(k-2)^{2} / 64\right\rfloor$. We have $3 t+m \leq k^{2} / 64-(k-2)^{2} / 64+1=(k-1) / 16+1=(k+15) / 16$.

Claim 6: We have $t+2 m \leq k-3$.

Proof of Claim 6: We just saw that $3 t+m \leq(k+15) / 16$. We use that $k \geq 20$.

Let $F \subset Q$ be a disjoint union of $t$ elements of type $\left|\mathcal{O}_{Q}(1,0)\right|$ and $m$ connected elements of $\left|\mathcal{O}_{Q}(2,0)\right|$. The scheme $F$ is a disjoint union of $t$ lines and $m$ 2-lines of $Q$ and hence $h^{0}\left(\mathcal{O}_{F}(k)\right)=(t+2 m)(k+1)$. Write $F=F_{1} \sqcup F_{2}$ with $F_{2}$ the union of the degree two connected components and set $G:=\left(F_{2}\right)_{\text {red }}$, $E:=F_{1} \sqcup 2 G$. We have $E \in L\left(t, 0, c-\left\lfloor(k-2)^{2} / 64\right\rfloor\right), E \cap Q=F$ and $\operatorname{Res}_{Q}(E)=G$. Set $\left.e:=\left\lfloor(k+1)(k+1-t-2 m)-6\left\lfloor(k-2)^{2} / 64\right\rfloor\right) / 3\right\rfloor$ and $f:=(k+1)(k+1-t-2 m)-6\left\lfloor(k-2)^{2} / 64\right\rfloor-3 e$. We have $0 \leq f \leq 2$.

Claim 7: We have $e \geq 2$.

Proof of Claim 7: Assume $e \leq 1$. Since $f \leq 2$, we get $(k+1)(k+1-t-$ $2 m)-6\left\lfloor(k-2)^{2} / 64\right\rfloor \leq 5$. Since $3 t+m \leq(k+15) / 16$, we have $t+2 m \leq(k+15) / 8$. Hence $(k+1)(k+1-t-2 m) \geq 7(k+1)(k-14) / 8>6\left\lfloor(k-2)^{2} / 64\right\rfloor+5$ for all $k \geq 20$.

Claim 8: We have $a \geq e+f$.

Proof of Claim 8: We have $4 a+(k+1) t+(3 k+1) c \geq\left(\begin{array}{c}k+3 \\ 3\end{array}\right)-3$. Since $3 t+c \leq\left\lfloor k^{2} / 64\right\rfloor$, we get $4 a \geq\left(\begin{array}{c}k+3 \\ 3\end{array}\right)-3-(3 k+1) k^{2} / 64$. Since $t+m>0$ and $f \leq 2$, we have $3 e+f \leq(k+1) k-6\left\lfloor(k-2)^{2} / 64\right\rfloor$. See the proof of Claim 3 .

By Claim 8 we have $a-e-f \geq 0$. Fix a general $Y \in L(0, a-e-f,\lfloor(k-$ $\left.2)^{2} / 64\right\rfloor$. The scheme $Y \cap Q$ is a general union of $2\left\lfloor(k-2)^{2} / 64\right\rfloor 2$-points of $Q$.

By Claim 7 we have $e \geq 0$. Fix a general $S \cup S^{\prime} \subset Q$ such that $\sharp(S)=e$, $\sharp\left(S^{\prime}\right)=f$ and $S \cup S^{\prime}$. Set $X^{\prime}:=Y \cup E \cup 2 S$. We have $X^{\prime} \in L(t, a-f, c)$. Hence to prove Theorem 1 it is sufficient to prove that a general union, $X^{\prime \prime}$, of $X^{\prime}$ and $f$ 2-points satisfies $h^{1}\left(\mathcal{I}_{X^{\prime \prime}}(k)\right)=0$. We have $3 e+f=(k+1)(k+1-$ 
$2 m-t)-6\left\lfloor(k-2)^{2} / 64\right\rfloor$. Since $t+2 m \leq k-3$ (Claim 5), [14, Proposition 5.2 and Theorem 7.2] gives $h^{i}\left(Q, \mathcal{I}_{(Y \cap Q) \cup\{2 S, Q\} \cup S^{\prime}}(k)(-F)\right)=0, i=0,1$. By the differential Horace lemma for 2-points ([1], [11, Lemma 5]) to prove that $h^{1}\left(\mathcal{I}_{X^{\prime \prime}}(k)\right)=0$ (and hence to prove this part of Theorem 1 in this case) it is sufficient to prove that $h^{1}\left(\mathcal{I}_{Y \cup G \cup S \cup\left\{2 S^{\prime}, Q\right\}}(k-2)\right)=0$.

Claim 9: $h^{1}\left(\mathcal{I}_{Y \cup G \cup\left\{2 S^{\prime}, Q\right\}}(k-2)\right)=0$.

Proof of Claim 9: It is sufficient to prove that $h^{1}\left(\mathcal{I}_{Y \cup G \cup 2 S^{\prime}}(k-2)\right)=0$. We have $h^{0}\left(\mathcal{O}_{Y \cup G \cup 2 S^{\prime}}(k-2)\right)=\left(\begin{array}{c}k+1 \\ 3\end{array}\right)-\beta-e+f \leq\left(\begin{array}{c}k+1 \\ 3\end{array}\right)$ (Claim 8). Since $\operatorname{deg}(G) \leq\left\lfloor k^{2} / 64\right\rfloor-\left\lfloor(k-2)^{2} / 64\right\rfloor$ and $\sharp\left(S^{\prime}\right)=f \leq 2$, to prove Claim 10 it is sufficient to apply the case $h=\operatorname{deg}(G)$ and $g=\sharp\left(S^{\prime}\right)$ of $H_{k-2}$.

Hence to conclude the proof part (i) of Theorem 1 in this case it is sufficient to prove the following claim.

Claim 10: $h^{0}\left(\mathcal{I}_{Y}(k-4)\right) \leq \beta$.

Proof of Claim 10: Take $M \in L\left(0, a-e-f,\left\lfloor(k-4)^{2} / 64\right\rfloor\right)$ such that $M \subseteq Y$. By the inductive assumption either $h^{0}\left(\mathcal{I}_{M}(k-4)\right)=0$ (and hence $\left.h^{0}\left(\mathcal{I}_{Y}(k-4)\right)=0\right)$ or $h^{1}\left(\mathcal{I}_{M}(k-4)\right)=0$. We may assume that the latter case occurs and hence $h^{0}\left(\mathcal{I}_{M}(k-4)\right)=\left(\begin{array}{c}k-1 \\ 3\end{array}\right)-4(a-e-f)-(3 k-11)\left\lfloor(k-4)^{2} / 64\right\rfloor$. We have $3 e+f+6\left\lfloor(k-2)^{2} / 64\right\rfloor=(k+1)^{2}-(k+1) t-2(k+1)\left(c-\left\lfloor(k-2)^{2} / 64\right\rfloor\right)$ and $4 a+(k+1) t+(3 k+1) c=\left(\begin{array}{c}k+3 \\ 3\end{array}\right)-\beta$. To prove Claim 10 it is sufficient to prove the inequality $4(e+f)+(k+1) t+(3 k+1)\left(c-\left\lfloor(k-4)^{2} / 64\right\rfloor\right)+$ $6\left\lfloor(k-2)^{2} / 64\right\rfloor \leq(k+1)^{2}+(k-1)^{2}=2 k^{2}+2$. Recall that $3 e+f+6\lfloor(k-$ $\left.2)^{2} / 64\right\rfloor+\left(t+2\left(c-\left\lfloor(k-2)^{2} / 64\right\rfloor\right)\right)=(k+1)^{2}$. Set $\eta:=\left\lfloor(k-2)^{2} / 64\right\rfloor-$ $\left\lfloor(k-4)^{2} / 64\right\rfloor$. We have $|\eta-(3 k-3) / 16|<2$. It is sufficient to prove that $(k-1)\left(c-\left\lfloor(k-2)^{2} / 64\right\rfloor\right)+3 f+6\left\lfloor(k-4)^{2} / 64\right\rfloor+(3 k-5) \eta \leq(k-1)^{2}$. We have $c-\left\lfloor(k-2)^{2} / 64\right\rfloor \leq\left\lfloor k^{2} / 64\right\rfloor-\left\lfloor(k-2)^{2} / 64\right\rfloor \leq(k-1) / 16$. We have $(k-1)^{2} / 16+6+3(k-4)^{2} / 32+(3 k-5)(3 k-3) / 64+(3 k-5) / 32 \leq(k-1)^{2}$

In this case we put all $t$ lines inside $Q$ and $Q$ contains $e \geq 2$ of the points in the support of the 2-points of our solution. Hence $H_{k}$ is proved in this case.

(ii) Assume $(k+1) t+4 a+(3 k+1) c \geq\left(\begin{array}{c}k+3 \\ 3\end{array}\right)$. Set $\beta^{\prime}:=\left(\begin{array}{c}k+3 \\ 3\end{array}\right)-(k+$ $1) t-4 a-(3 k+1) c$. By the case $\beta=0$ of step (i) we may assume $\beta^{\prime}>0$. Since $(k+1) t+(3 k+1) c \leq\left(\begin{array}{c}k+3 \\ 3\end{array}\right)$, we have $a>0$. Hence decreasing if necessary $a$ we may assume $0<\beta^{\prime} \leq 3$. Repeat the proofs of step (i), avoiding step (i1.1).

(iii) Assume $k \leq 19$. We have $3 t+c \leq 0$ if $k \leq 7,3 t+c \leq 1$ if $k \leq 11$, $3 t+c \leq 2$ if $k=12,13,3 t+c \leq 3$ if $k=14,15,3 t+c \leq 4$ if $k=16,17,3 t+c \leq 5$ if $k=19$. Since the case $c=0$ is true by [4], we may assume $c>0$. Hence $t=0$. All cases are done as in step (i2) taking as $G$ the union of $c$ general connected elements of $\left|\mathcal{O}_{Q}(2,0)\right|$. 


\section{Proofs of Propositions 1 and 2}

Set $u_{r, k}:=\left\lceil\left(\begin{array}{c}r+k \\ r\end{array}\right) /(k+1)\right\rceil$ and $v_{r, k}:=(k+1) u_{r, k}-\left(\begin{array}{c}r+k \\ r\end{array}\right)$. We have $0 \leq v_{r, k} \leq k$ and $(k+1) u_{r, k}-v_{r, k}=\left(\begin{array}{c}r+k \\ r\end{array}\right)$.

Lemma 36. We have $u_{r, k}-u_{r, k-1} \geq 2 k$ if either $r=4$ and $k \geq 12$ or $r \geq 5$ and $k \geq 2$. We have $u_{5,1}=3, v_{5,1}=0, u_{5,2}=7, v_{5,2}=0, u_{5,3}=14$, $v_{5,3}=0, u_{4,1}=3, v_{4,1}=1, u_{4,2}=5, v_{4,2}=0, u_{4,3}=9, v_{4,3}=1, u_{4,4}=14$, $v_{4,4}=0, u_{4,5}=26, v_{4,5}=4, u_{4,6}=30, v_{4,6}=0, u_{4,7}=42, v_{4,7}=6, u_{4,8}=55$, $v_{4,8}=0, u_{4,9}=72, v_{4,9}=5, u_{4,10}=91, v_{4,10}=0, u_{4,11}=114, v_{4,11}=3$.

Proof. Since $u_{r, k}-u_{r, k-1} \geq\left(\begin{array}{c}r+k \\ r\end{array}\right) /(k+1)-\left(\begin{array}{c}r+k-1 \\ r\end{array}\right) / k-1$, it is sufficient to check when $\left(\begin{array}{c}r+k-1 \\ r\end{array}\right)(r-1) \geq k(k+1)(2 k+1)$. The last inequality is true if either $r=4$ and $k \geq 12$ or $r=5$ and $k \geq 4$ or $r \geq 6$ and $k \geq 2$. The explicit values are easily computed.

Lemma 37. For all integers $r \geq 4$ and $k \geq 2$ we have $u_{r, k}-2 v_{r, k} \geq 0$ and $h^{i}\left(\mathcal{I}_{X}(k)\right)=0, i=0,1$, for a general union $X \subset \mathbb{P}^{r}$ of $u_{r, k}-2 v_{r, k}$ lines and $v_{r, k}$ reducible conics whose singular point is contained in $H$.

Proof. The lemma says that Assertion $H_{k, r}^{\prime}$ of [13, page 182] is true. Use $[13, \S 3]$ (if $k$ is low and $r=4,5$, one can also use Lemma 36).

Lemma 38. Fix integers $k \geq 3 r \geq 4, t \geq 0$ and $e$ such that $0 \leq e \leq k$ and $t(k+1)+(2 k+1) e \leq\left(\begin{array}{c}r+k \\ r\end{array}\right)$. Then $h^{1}\left(\mathcal{I}_{X}(k)\right)=0$ for a general union $X \subset \mathbb{P}^{r}$ of $t$ lines and e reducible conics whose singular point is contained in $H$.

Proof. Set $\beta:=\left(\begin{array}{c}r+k \\ r\end{array}\right)-(k+1) t-e(2 k+1)$. Increasing if necessary $t$ we may assume that $0 \leq \beta \leq k$. We have $t=u_{r, k}-2 e$ if $e \geq v_{r, k}$ and $t=u_{r, k}-2 e-1$ if $e<v_{r, k}$. First assume $e \leq v_{r, k-1}$. Let $Y \subset \mathbb{P}^{r}$ be a general union of $u_{r, k-1}-2 v_{r, k-1}$ lines and $v_{r, k-1}$ reducible conics with singular points contained in $H$. Lemma 37 gives $h^{i}\left(\mathcal{I}_{Y}(k-1)\right)=0, i=0,1$. For a general $Y$ we may assume that $Y \cap H$ is a general union of $u_{r, k-1}-2 v_{r, k-1}$ points and $v_{r, k-1}$ tangent vectors of $H$. Write $Y=Y_{1} \sqcup Y_{2}$ with $Y_{2}$ the union of the degree two connected components of $H$. Since $e \leq k$ and $k \geq r$, Lemma 36 implies $u_{r, k-1}-2 v_{r, k-1} \geq e-v_{r, k-1}$. Hence we may write $Y_{1}=Y^{\prime} \sqcup Y^{\prime \prime}$ with $\operatorname{deg}\left(Y^{\prime}\right)=e-v_{r, k-1}$. For each connected component $L$ of $Y^{\prime \prime}$ take a general line $R_{L} \subset H$ through the point $L \cap H$. Set $G:=\cup_{L \subseteq Y^{\prime}} R_{L}$. Since $t \geq u_{r, k}-v_{r, k}$, Lemma 36 gives $t-\operatorname{deg}(Y) \geq e-v_{r, k-1}$. Let $F \subset H$ be a general union of $t-\operatorname{deg}(Y)-\operatorname{deg}(G)$ lines. Set $X:=Y \cup G \cup F$. $X$ is a disjoint union of $t$ lines and $e$ reducible conics with singular point contained in $H$. Hence to prove 
the lemma in this case it is sufficient to prove that $h^{1}\left(\mathcal{I}_{X}(k)\right)=0$. By [13] we have $h^{1}\left(H, \mathcal{I}_{F \cup G}(k)\right)=0$. Since $Y \cap H$ is a general union of points and tangent vectors and $\beta \geq 0$, we get $h^{1}\left(H, \mathcal{I}_{X \cap H}(k)\right)=0$, by the case $q^{\prime \prime}=t=0$, $d=v_{r, k-1}$ of $\left[13, H_{k-1, r}^{\prime \prime}\right]$. Now assume $v_{r, k-1}>e$. Let $E \subset H$ be a general union of $t-u_{r, k-1}$ lines. Write $Y_{2}=U \sqcup V$ with $U$ union of $e$ reducible conics and $V$ union of $v_{r, k-1}-e$ reducible conics. For each $P \in \operatorname{Sing}(V)$ fix a 3-dimensional space $W_{P} \subset \mathbb{P}^{r}$ containing the connected component $D_{P}$ of $V$ containing $P$ and set $w_{P}:=\left\{2 P, W_{P}\right\}$. Set $\mathbf{w}:=\cup_{P \in \operatorname{Sing}(V)} w_{P}$. The scheme $X^{\prime}:=E \cup Y \cup \mathbf{w} \cup Z$ is a flat limit of a family of disjoint unions of $U$ and $t$ lines. By the semicontinuity theorem for cohomology it is sufficient to prove that $h^{1}\left(\mathcal{I}_{X^{\prime}}(k)\right)=0$. We have $\operatorname{Res}_{H}\left(X^{\prime}\right)=Y$ and hence $h^{1}\left(\mathcal{I}_{\operatorname{Res}_{H}\left(X^{\prime}\right)}(k)\right)=0$. The scheme $X^{\prime} \cap H$ is a general union of $E$, $e$ tangent vectors and $v_{r, k-1}-e$ degree 3 zero-dimensional schemes called triple points in [13]. Apply [13, $H_{k, r}^{\prime \prime}$ ] with $q^{\prime \prime}=0, d=e$ and $t=v_{r, k-1}-e$ and get $h^{1}\left(H, \mathcal{I}_{X^{\prime} \cap H}(k)\right)=0$. Apply the Castelnuovo's inequality.

Proof of Proposition 1: We fix $z \in \mathbb{N}$ and a zero-dimensional scheme $Z \subset \mathbb{P}^{r}$ with $\operatorname{deg}(Z) \leq z$. We take any hyperplane $H \subset \mathbb{P}^{r}$ such that $Z \cap H=\emptyset$. Hence in $H$ we only need the case $z=0$. If $r=4$, then we use [4]. By [6] we may assume $r \geq 6$. Assume that Proposition 1 for $z=0$ is true in $\mathbb{P}^{r-1}$ (if $r=4$ we need [6] instead of the inductive assumption) and call $\beta_{r-1,0}$ the corresponding integer (we may take $\beta_{r-1,0}=0$ ([4], [6], [7]), but we do not need it). Increasing if necessary $\beta_{r-1,0}$ we may assume that $\beta_{r-1,0} \geq r$. Set $\beta_{r, z}:=\left(\begin{array}{c}4 r+\beta_{r-1,0}+z \\ r\end{array}\right)$. Fix $(t, a) \in \mathbb{N}^{2}$ and let $k$ be its critical value with respect to $z$, i.e. the first integer $k>0$ such that $z+t(k+1)+(r+1) a \leq\left(\begin{array}{c}r+k \\ r\end{array}\right)$. By the CastelnuovoMumford's lemma a scheme $X=W \sqcup Z$ with $W \in L(t, a, 0 ; r)$ has maximal rank if and only if $h^{1}\left(\mathcal{I}_{X}(k)\right)=0$ and $h^{0}\left(\mathcal{I}_{X}(k-1)\right)=0$. Since $L(t, a, 0 ; r)$ is irreducible, by the semicontinuity theorem for cohomology it is sufficient to find $A, B \in L(t, a, 0 ; r)$ such that $A \cap Z=B \cap Z=\emptyset, h^{1}\left(\mathcal{I}_{A \cup Z}(k)\right)=0$ and $h^{0}\left(\mathcal{I}_{A \cup Z}(k-1)\right)=0$. Since $\beta_{r, z} \geq\left({ }^{4 r+\beta_{r-1,0}}\right)$, we have $k \geq 3 r+\beta_{r-1,0}+z$. Hence for this fixed choice of $\beta_{r, z}$ Proposition 1 is trivially true if $k<3 r+\beta_{r-1,0}+z$ (there is no such datum $(t, a)$ ). Hence we use induction on $k$.

(a) In this step we prove the existence of $A$. Set $\beta:=\left(\begin{array}{c}r+k \\ r\end{array}\right)-t(k+1)-$ $(r+1) a-z$.

(a1) Assume $(k+1) t+\beta \geq\left(\begin{array}{c}r+k-1 \\ r-1\end{array}\right)$. Let $x$ be the minimal integer such that $(t-x)+x(k+1)+\beta \geq\left(\begin{array}{c}r+k-1 \\ r-1\end{array}\right)$ (it exists, because $k>0$ ). By assumption we have $x \leq t$. Set $\gamma:=\left(\begin{array}{c}r+k-1 \\ r-1\end{array}\right)-\beta-(t-x)-x(k+1)$. The minimality of the integer $x$ implies that $\gamma \leq k$. Since $(k+1) t+\beta \leq\left(\begin{array}{c}r+k \\ r\end{array}\right)$, we have $x \geq 0$. Since $k \geq 3 r$, we also have $x \geq 2 k$. Hence $x \geq 2 \gamma$. Let 
$E \subset H$ be a general union of $x-2 \gamma$ lines and $\gamma$ reducible conics. Write $E=E_{1} \sqcup E_{2}$ with $E_{i}$ the union of the degree $i$ connected components of $E$. For each $P \in \operatorname{Sing}\left(E_{2}\right)$ let $V_{P} \subset \mathbb{P}^{r}$ be a 3-dimensional linear subspace such that $V_{P} \cap H$ is a plane spanned by the connected component of $E_{2}$ containing $P$. Set $v_{P}:=\left\{2 P, V_{P}\right\}$ and $\mathbf{v}:=\cup_{P \in \operatorname{Sing}\left(E_{2}\right)} v_{P}$. Notice that $(E \cup \mathbf{v}) \cap H=E$ (schemetheoretic intersection). Fix a general $Y \in L(t-x, a, 0 ; r)$. Since $Y$ is general, no component of $Y_{\text {red }}$ is contained in $H, Z \cap Y=\emptyset, Y \cap E=\emptyset$ and $Y \cap H$ is a general union of $t-x$ general points of $H . E_{2} \cup \mathbf{v}$ is a disjoint union of $\gamma$ sundial in the sense of and hence it is a flat limit of a family of disjoint unions of $2 \gamma$ lines ([13, Example 2.1.1], [9]). Hence $X:=Z \cup Y \cup E \cup \mathbf{v}$ is a flat limit of a family of elements of $L(t, a, 0 ; r)$. Hence to prove the existence of $A$ in this case it is sufficient to prove that $h^{1}\left(\mathcal{I}_{X}(k)\right)=0$. We have $\operatorname{Res}_{H}(X)=Z \cup Y \cup \operatorname{Sing}\left(E_{2}\right)$. Since $\sharp\left(\operatorname{Sing}\left(E_{2}\right)\right)=\gamma$ and $Y \cap E=Y \cap Z=\emptyset$, the definitions of $\beta$ and $\gamma$ give $h^{0}\left(\mathcal{O}_{Z \cup Y \cup \operatorname{Sing}\left(E_{2}\right)}(k-1)\right)=\left(\begin{array}{c}r+k-1 \\ r\end{array}\right)$. The inductive assumption on $k$ gives $h^{1}\left(\mathcal{I}_{Z \cup Y}(k-1)\right)=0$ and hence $h^{0}\left(\mathcal{I}_{Z \cup Y}(k-1)\right)=\gamma$. Since $\operatorname{Sing}\left(E_{2}\right)$ is a general subset of $H$ with cardinality $\gamma$ and $\operatorname{Res}_{H}(Y)=Y$, to prove that $h^{i}\left(\mathcal{I}_{Z \cup Y \cup \operatorname{Sing}\left(E_{2}\right)}(k-1)\right)=0$, it is sufficient to check that $h^{0}\left(\mathcal{I}_{Z \cup Y}(k-2)\right)=0$. This is true by the inductive assumption on $k$. Since $\beta \geq 0$ and $r \geq 5$, Lemma 36 gives $h^{1}\left(H, \mathcal{I}_{E}(k)\right)=0$ and $h^{0}\left(H, \mathcal{I}_{E}(k)\right)=\beta+t-x$. Since $Y \cap H$ is a general subset of $H$ with cardinality $t-x$ and $\beta \geq 0$, we get $h^{1}\left(H, \mathcal{I}_{X \cap H}(k)\right)=0$. The Castelnuovo's sequence gives $h^{1}\left(\mathcal{I}_{X}(k)\right)=0$.

(a2) Assume $(k+1) t+\beta<\left(\begin{array}{c}r+k-1 \\ r-1\end{array}\right)$.

(a2.1) First assume $(k+1) t+r \beta_{r-1,0} \leq\left(\begin{array}{c}r+k-1 \\ r-1\end{array}\right)$. Set $e:=\left\lfloor\left(\left(\begin{array}{c}r+k-1 \\ r-1\end{array}\right)-(k+\right.\right.$ $\left.\left.1) t-r \beta_{r-1,0}\right) / r\right\rfloor$ and $f:=\left(\begin{array}{c}r+k-1 \\ r-1\end{array}\right)-(k+1) t-r \beta_{r-1,0}-r e$. We have $0 \leq f \leq r-1$. Let $M \subset H$ be a general union of $t$ lines. Fix a general $S \cup S^{\prime} \subset H$ such that $\sharp(S)=\beta_{r-1,0}+e, \sharp\left(S^{\prime}\right)=f$ and $S \cap S^{\prime}=\emptyset$. We have $h^{0}\left(\mathcal{O}_{M \cup\{2 S, H\} \cup S^{\prime}}(k)\right)=$ $\left(\begin{array}{c}r+k-1 \\ r-1\end{array}\right)-\beta$. Since $e \geq 0$, we have $\sharp(S) \geq \beta_{r-1,0}$. Hence the inductive assumption on $r$ (using [4] if $r-1=3$ ) gives $h^{1}\left(H, \mathcal{O}_{M \cup\{2 S, H\} \cup S^{\prime}}(k)\right)=0$.

Claim 1: We have $a \geq \beta_{r-1, z}+e+f$.

Proof of Claim 1: We have $(k+1) t+r\left(\beta_{r-1,0}+e\right)+f=\left(\begin{array}{c}r+k-1 \\ r-1\end{array}\right)-\beta$ and $(k+1) t+(r+1) a=\left(\begin{array}{c}r+k \\ r\end{array}\right)-\beta$. Use that $f \leq r$ and that $k \geq 3 r$.

Since $\beta_{r, z} \geq \beta_{r-1, z}+r-1 \geq \beta_{r-1,0}+f$, we have $a \geq \beta_{r-1,0}+e+f$.

By Claim 1 we have $a \geq \beta_{r, z}+e+f$. Fix a general $J \in L(0, a-e-$ $f, 0 ; r)$. By the differential Horace lemma for 2-points ([1], [11, Lemma 5], [2] in characteristic $\neq 2$ ) to prove that a general union $X^{\prime \prime}$ of $Z \cup J \cup S$ and $f 2$-points satisfies $h^{1}\left(\mathcal{I}_{X^{\prime \prime}}(k-1)\right)=0$ (and hence to prove the proposition in this case) it is sufficient to prove that $h^{1}\left(\mathcal{I}_{Z \cup J \cup S \cup\left\{2 S^{\prime}, H\right\}}(k-1)\right)=0$.

Claim 2: $h^{1}\left(\mathcal{I}_{Z \cup J \cup\left\{2 S^{\prime}, H\right\}}(k-1)\right)=0$. 
Proof of Claim 2: Set $U^{\prime}:=J \cup 2 S^{\prime}$. Since $\sharp\left(S^{\prime}\right)=f \leq r-1$ and any $r$ points of $\mathbb{P}^{r}$ are contained in a hyperplane, $U^{\prime}$ may be considered as a general union of $a-e-\beta_{r-1,0} 2$-points. Since $\beta_{r-1,0} \geq r$, we have $\sharp(S) \geq \sharp\left(S^{\prime}\right)$. Since $h^{0}\left(\mathcal{O}_{Z \cup Y_{3} \cup S \cup\left\{2 S^{\prime}, H\right\}}(k-1)\right)=\left(\begin{array}{c}r+k-1 \\ r\end{array}\right)$, we get $h^{0}\left(\mathcal{O}_{Z \cup U^{\prime}}(k-1)\right) \leq\left(\begin{array}{c}r+k-1 \\ r\end{array}\right)$. Hence $h^{1}\left(\mathcal{I}_{Z \cup U^{\prime}}(k-1)\right)=0$ by the inductive assumption on the critical value. Since $Z \cup J \cup\left\{2 S^{\prime}, H\right\} \subseteq Z \cup U^{\prime}$ and $Z \cup U^{\prime}$ and $Z \cup J \cup\left\{2 S^{\prime}, H\right\}$ have the same positive -dimensional connected components, the restriction map $H^{0}\left(\mathcal{O}_{Z \cup U^{\prime}}(k-\right.$ 1) $\rightarrow H^{0}\left(\mathcal{O}_{Z \cup W}(k-1)\right)$ is surjective. Hence $h^{1}\left(\mathcal{I}_{Z \cup J \cup\left\{2 S^{\prime}, H\right\}}(k-1)\right)=0$, proving Claim 2.

By the definitions of $\beta$, e and $f$ we have $h^{0}\left(\mathcal{O}_{Z \cup J \cup S \cup\left\{2 S^{\prime}, H\right\}}(k-1)\right)=$ $\left(\begin{array}{c}r+k-1 \\ r\end{array}\right)$. Since $J$ is general, we have $\operatorname{Res}_{H}\left(Z \cup J \cup\left\{2 S^{\prime}, H\right\}\right)=Z \cup J$. Since $S$ is general in $H$, Claim 2 shows that to prove that $h^{1}\left(\mathcal{I}_{Z \cup J \cup S \cup\left\{2 S^{\prime}, H\right\}}(k-1)\right)=0$ (and hence to prove the proposition in this case) it is sufficient to prove that $h^{0}\left(\mathcal{I}_{J}(k-2)\right)=0$. Since $k-2 \geq 5$ and $J$ is general, the inductive assumption on $k$ shows that it is sufficient to check that $(r+1)\left(a-\beta_{r-1,0}-e-f\right) \geq\left(\begin{array}{c}r+k-2 \\ r\end{array}\right)$. By the definitions of $\beta, e$ and $f$ we have we have $(r+1)\left(a-\beta_{r-1,0}-e-f\right)+\beta_{r-1,0}+$ $e+r f=\left(\begin{array}{c}r+k-1 \\ r\end{array}\right)$. Hence it is sufficient to check that $\beta_{r-1,0}+e+r f \leq\left(\begin{array}{c}r+k-2 \\ r-1\end{array}\right)$. Since $t \geq 0$, we have $z+r\left(\beta_{r-1,0}+e\right)+f \leq\left(\begin{array}{c}r+k-1 \\ r-1\end{array}\right)$. Use that $k>r$.

(a2.2) Now assume $(k+1) t+r \beta_{r-1,0}>\left(\begin{array}{c}r+k-1 \\ r-1\end{array}\right)$. Let $t^{\prime}$ be the maximal integer such that $(k+1) t^{\prime}+r \beta_{r-1,0} \leq\left(\begin{array}{c}r+k-1 \\ r-1\end{array}\right)$. By assumption we have $t^{\prime}<t$.

Claim 3: We have $t^{\prime} \geq 0$.

Proof of Claim 3: By the definition of $\beta_{r, z}$ we have $k \geq \beta_{r-1,0}$. Hence the inequality $t^{\prime} \geq 0$ is obvious.

Set $e^{\prime}:=\left\lfloor\left(\left(\begin{array}{c}r+k-1 \\ r-1\end{array}\right)-(k+1) t^{\prime}-r \beta_{r-1,0}\right) / r\right\rfloor$ and $f^{\prime}:=\left(\begin{array}{c}r+k-1 \\ r-1\end{array}\right)-(k+$ 1) $t-r \beta_{r-1,0}-r e^{\prime}$. We have $0 \leq f^{\prime} \leq r-1$. Let $M^{\prime} \subset H$ be a general union of $t$ lines. Fix a general $S_{1} \cup S_{1}^{\prime} \subset H$ such that $\sharp\left(S_{1}\right)=\beta_{r-1,0}+e^{\prime}$, $\sharp\left(S_{1}^{\prime}\right)=f^{\prime}$ and $S_{1} \cap S_{1}^{\prime}=\emptyset$. We have $h^{0}\left(\mathcal{O}_{M^{\prime} \cup\left\{2 S_{1}, H\right\} \cup S_{1}^{\prime}}(k)\right)=\left(\begin{array}{c}r+k-1 \\ r-1\end{array}\right)-\beta$. Since $e^{\prime} \geq 0$, we have $\sharp\left(S_{1}\right) \geq \beta_{r-1,0}$. Hence the inductive assumption on $r$ (or [4], [6], [7]) gives $h^{1}\left(H, \mathcal{I}_{M^{\prime} \cup\left\{2 S_{1}, H\right\} \cup S_{1}^{\prime}}(k)\right)=0$. As in the proof of Claim 1 we see that $a \geq e^{\prime}+f^{\prime}$. Fix a general $Y_{1} \in L\left(t-t^{\prime}, a-e^{\prime}-f^{\prime}, 0 ; r\right)$. By the differential Horace lemma for 2-points ([1], [11, Lemma 5]) to prove that a general union $X_{2}$ of $Z \cup Y_{1} \cup S_{1}$ and $f$ 2-points satisfies $h^{1}\left(\mathcal{I}_{X_{2}}(k-1)\right)=0$ (and hence to prove the existence of $A$ in this case) it is sufficient to prove that $h^{1}\left(\mathcal{I}_{Z \cup Y_{1} \cup S_{1} \cup\left\{2 S_{1}, H\right\}}(k-1)\right)=0$. Assume for the moment $h^{1}\left(\mathcal{I}_{Y_{1} \cup\left\{2 S_{1}^{\prime}, H\right\}}(k-\right.$ $1))=0$. We have $h^{0}\left(\mathcal{I}_{Z \cup Y_{1} \cup S_{1} \cup\left\{2 S_{1}^{\prime}, H\right\}}(k-1)\right)=\left(\begin{array}{c}r+k-1 \\ r\end{array}\right)$. Since $S_{1}$ is general in $H$, to prove that $h^{1}\left(\mathcal{I}_{Z \cup Y_{1} \cup S_{1} \cup\left\{2 S_{1}^{\prime}, H\right\}}(k-1)\right)=0$ it is sufficient to prove that $h^{0}\left(\mathcal{I}_{Z \cup Y_{1}}(k-2)\right)=0$. See step $(\mathrm{a} 2.2 .2)$.

Claim 4: We have $k\left(t-t^{\prime}\right)+r \beta_{r-1,0} \leq\left(\begin{array}{c}r+k-2 \\ r-1\end{array}\right)$. 
Proof of Claim 4: The maximality property of the integer $t^{\prime}$ gives $(k+$ 1) $t^{\prime}+r \beta_{r-1,0} \geq\left(\begin{array}{c}r+k-1 \\ r-1\end{array}\right)-k$. Since $(k+1) t+\beta<\left(\begin{array}{c}r+k-1 \\ r-1\end{array}\right)$, we get $(k+$ 1) $\left(t-t^{\prime}\right)+\beta-r \beta_{r-1,0} \leq k-1$. Since $\beta \geq 0$, it is sufficient to check that $2 r \beta_{r-1,0}+k-1 \leq\left(\begin{array}{c}r+k-2 \\ r-1\end{array}\right)$. This is true, because our assumption on $\beta_{r}$ implies $k \geq 3 r+\beta_{r-1,0}$.

(a2.2.1) Now we check that $h^{1}\left(\mathcal{I}_{Z \cup Y_{1} \cup\left\{2 S_{1}^{\prime}, H\right\}}(k-1)\right)=0$. By the semicontinuity theorem for cohomology it is sufficient to prove this $h^{1}$-vanishing for a specialization $Y_{2}$ of $Y_{1}$. Set $e^{\prime \prime}:=\left\lfloor\left(\left(\begin{array}{c}r+k-2 \\ r-1\end{array}\right)-k\left(t-t^{\prime}\right)-r f^{\prime}\right) / r\right\rfloor$. Since $f^{\prime} \leq r$, we have $f^{\prime} \leq \beta_{r-1,0}$. Claim 4 gives $e^{\prime \prime} \geq \beta_{r-1}$. Fix a general union $E \subset H$ of $t-t^{\prime}$ lines. Fix a general $S_{2} \cup S_{2}^{\prime} \subset H$ such that $\sharp\left(S_{2}\right)=e^{\prime \prime}-f^{\prime}, \sharp\left(S_{2}^{\prime}\right)=f^{\prime \prime}$ and $S_{2} \cap S_{2}^{\prime}=\emptyset$. We have $h^{0}\left(\mathcal{O}_{E \cup\left\{2 S_{1}^{\prime}, H\right\} \cup\left\{2 S_{2}, H\right\} \cup S_{2}^{\prime}}(k-1)\right)=\left(\begin{array}{c}r+k-2 \\ r-1\end{array}\right)$. Since $\sharp\left(S_{1}^{\prime}\right)+\sharp\left(S_{2}\right) \geq \beta_{r-1,0}$, either the inductive assumption on $r$ or [4], [6], [7] give $h^{i}\left(H, \mathcal{I}_{E \cup\left\{2 S_{1}^{\prime}, H\right\} \cup\left\{2 S_{2}, H\right\} \cup S_{2}^{\prime}}(k-1)\right)=0, i=0,1$. Assume for the moment $e-e^{\prime}-e^{\prime \prime}-f^{\prime \prime} \geq 0$. In this case we take as a specialization of $Y_{1}$ a general union $Y_{2}$ of $f^{\prime \prime} 2$-points and $W^{\prime}:=W \cup 2 S_{2}$ with $W$ general in $L\left(0, a-e^{\prime}-e^{\prime \prime}-f^{\prime \prime}, 0 ; r\right)$. By the differential Horace lemma for 2-points ([1], $\left[11\right.$, Lemma 5]) to prove that $h^{1}\left(\mathcal{I}_{Z \cup Y_{2} \cup\left\{2 S_{1}^{\prime}, H\right\}}(k-1)\right)=0$ it is sufficient to prove that $h^{1}\left(\mathcal{I}_{Z \cup W \cup S_{2} \cup\left\{2 S_{2}^{\prime}, H\right\}}(k-2)\right)=0$. See the last part of step (a1) with $k-2$ instead of $k-1$. Now assume $a-e-e^{\prime}-e^{\prime \prime}-f^{\prime \prime}<0$. If $a-e-e^{\prime}-e^{\prime \prime} \geq 0$, take as a specialization of $Y_{1}$ a general union of $2 S_{2}$ and $a-e-e^{\prime}$ If $a-e-e^{\prime}-e^{\prime \prime}<0$, take $S_{3} \subset S_{2}$ with $\sharp\left(S_{3}\right)=a-e-e^{\prime}$ and take $2 S_{3}$ as a specialization of $Y_{1}$.

(a2.2.2) Now we check that $h^{0}\left(\mathcal{I}_{Z \cup Y_{1}}(k-2)\right)=0$. We repeat step (a2.2.1), i.e. we specialize the union of the lines of $Y_{1}$ to a general union $E \subset H$ of $t-t^{\prime}$ lines.

(b) In this step we prove the existence of $B$. Instead of $\beta$ we use the integer $\beta^{\prime}:=z+k t+(r+1) a-\left(\begin{array}{c}r+k-1 \\ r\end{array}\right)$. The definition of the integer $k$ gives $\beta^{\prime}>0$. Decreasing if necessary $t$ we may assume that either $t=0$ or $\beta^{\prime} \leq k-1$. We use $k-1$ instead of $k, k-2$ instead of $k-1$ and $k-3$ instead of $k-2$. In this step we use the assumption $k-3 \geq 5$ and that $k-1 \geq 3 r$ (as in the proof of Claim 1).

Proof of Proposition 2: We could easily modify the proof of Proposition 1, but we prefer to use the following quotations. Fix $(t, a) \in \mathbb{N}^{2}$ and a general $X \in$ $L(t, a, 0 ; r)$. If $a \geq \beta_{r, z}$, then $X$ has maximal rank by Proposition 1 . Hence we only need to test finitely many integers $a$, say $0 \leq a<\beta_{r, z}$. For any such $a$ fix any union $Z_{a} \subset \mathbb{P}^{r}$ of $a$ different 2-points. Use [3] for each of these schemes.

Proposition 3. Fix integers $r \geq 4, k \geq 6$ and $\left(t, c, a, t^{\prime}, a^{\prime}\right) \in \mathbb{N}^{5}$. Assume $t^{\prime}+t(k+1)+r a+((r-1) k+1) c \leq\left(\begin{array}{c}k+r-1 \\ r-1\end{array}\right),(r+1)\left(t+t^{\prime}\right)(k-1)+(r+1) a^{\prime} \leq$ 
$\left(\begin{array}{c}r+k-2 \\ r\end{array}\right), h^{1}\left(\mathbb{P}^{r-1}, \mathcal{I}_{F}(k)\right)=0$ for a general $F \in L(t, a, c ; r-1)$. If $\left(t^{\prime}, a^{\prime}\right) \neq 0$, then assume $k \geq 7$. Then $h^{1}\left(\mathcal{I}_{Z \cup X}(k)\right)=0$ for a general $X \in L\left(t+t^{\prime}, a+a^{\prime}, c ; r\right)$.

Proof. Fix a hyperplane $H \subset \mathbb{P}^{r}$. Let $E \subset \mathbb{P}^{r}$ be a general union of $t$ lines contained in $H, a$ 2-points whose support in $H$ and $c$ 2-lines whose support is contained in $H$. Set $F:=E \cap H$. Write $E=E_{1} \sqcup 2 S \sqcup 2 A$ with $E_{1}$ union of $t$ lines of $H, S$ a general subset of $H$ with cardinality $a$ and $A$ a general union of $c$ lines of $H$. Fix a general $Y \in L\left(t^{\prime}, a^{\prime}, 0 ; r\right)$. For a general $Y$ we have $Y \cap E=\emptyset$ and $Y \cap H$ is a general union of $t^{\prime}$ points of $H$. Set $W:=Y \cup E$. We have $\operatorname{Res}_{H}(W)=Y \cup A \cup S$. By the semicontinuity theorem for cohomology it is sufficient to prove that $h^{1}\left(\mathcal{I}_{W}(k)\right)=0$. Since $F$ is general in $L(t, a, c ; r-1)$, we have $h^{1}\left(H, \mathcal{I}_{F}(k)\right)=0$ ([4] if $r=4$, [6] if $r=5,6$ and [7] if $r \geq 7)$. Since $t^{\prime} \leq\left(\begin{array}{c}r+k-1 \\ r-1\end{array}\right)-t(k+1)-r a-((r-1) k+1) c=h^{0}\left(H, \mathcal{I}_{F}(k)\right)$, we get $h^{1}\left(H, \mathcal{I}_{H \cap W}(k)\right)=0$. Hence by the Castelnuovo's sequence it is sufficient to prove that $h^{1}\left(\mathcal{I}_{Y \cup A \cup S}(k-1)\right)=0$.

(a) Assume for the moment $\left(t^{\prime}, a^{\prime}\right)=(0,0)$, i.e. $Y=\emptyset$. Since $A \cup S \subset H$, we have $h^{1}\left(\mathcal{I}_{A \cup S}(k-1)\right)=h^{1}\left(H, \mathcal{I}_{A \cup S}(k-1)\right)$. Since $A$ is a general union of $c$ lines of $H$, it has maximal rank ([13]). Since $S$ is general in $H$, to conclude in this case it is sufficient to check that $k c+a \leq\left(\begin{array}{c}r+k-2 \\ r-1\end{array}\right)$. We have $r a+((r-1) k+1) c \leq$ $\left(\begin{array}{c}r+k-1 \\ r-1\end{array}\right)$ and $\left(\begin{array}{c}r+k-1 \\ r-1\end{array}\right) /\left(\begin{array}{c}r+k-2 \\ r-1\end{array}\right)=(r+k-1) /(k-2)<r-1$.

(b) Assume $\left(t^{\prime}, a^{\prime}\right) \neq(0,0)$. In this case if $c>r / 2$ the scheme $Y \cup A$ is not a general element of $L\left(t^{\prime}+c, a^{\prime}, 0 ; r\right)$ and hence we cannot just [6] and [7] to get that $L\left(t^{\prime}+c, a^{\prime}, 0 ; r\right)$ has maximal rank. Since $k-2 \geq 5$ and $(r+1)(t+$ $\left.t^{\prime}\right)(k-1)+(r+1) a^{\prime} \leq\left(\begin{array}{c}r+k-2 \\ r\end{array}\right)$, we have $h^{1}\left(\mathcal{I}_{Y}(k-2)\right)=0$ ([6], [7]). Since $h^{1}\left(H, \mathcal{I}_{A \cup S}(k-1)\right)=0$ by step (a), it is sufficient to apply the Castelnuovo's sequence.

\section{Acknowledgements}

The author was partially supported by MIUR and GNSAGA of INdAM (Italy).

\section{References}

[1] J. Alexander and A. Hirschowitz, Un lemme d'Horace différentiel: application aux singularité hyperquartiques de $\mathbb{P}^{5}$, J. Alg. Geom. 1 (1992), $411-426$. 
[2] J. Alexander and A. Hirschowitz, An asymptotic vanishing theorem for generic unions of multiple points, Invent. Math. 140 (2000), 303-325.

[3] E. Ballico, Postulation of a union $X \subset \mathbb{P}^{r}, r \geq 4$, of a given zerodimensional scheme and several general lines, ISRN Geometry Volume 2013, Article ID 391721, 6 pages http://dx.doi.org/10.1155/2013/391721

[4] E. Ballico, Postulation of general unions of lines and multiplicity two points in $\mathbb{P}^{3}$, Hindawi Publishing Corporation ISRN Geometry Volume 2014, Article ID 120850, 7 pages http://dx.doi.org/10.1155/2014/120850

[5] E. Ballico, Postulation in projective spaces of a union of a low dimensional scheme and general rational curves or general unions of lines, Gulf J. Math. (to appear).

[6] E. Ballico, Postulation of general unions of lines and multiplicity two points in $\mathbb{P}^{r}, r \leq 5$, preprint.

[7] E. Ballico, Postulation of general unions of lines and double points in a higher dimensional projective space, preprint.

[8] A. Bernardi, M. V. Catalisano, A. Gimigliano and M. Idà, Secant varieties to osculating varieties of Veronese embeddings of $\mathbb{P}^{n}$. J. Algebra 321 (2009), no. 3, 982-1004.

[9] E. Carlini, M. V. Catalisano and A. V. Geramita, 3-dimensional sundials, Cent. Eur. J. Math. 9 (2011), no. 5, 949-971.

[10] E. Carlini, M. V. Catalisano and A. V. Geramita, Reduced and nonreduced linear spaces: lines and points, arXiv: 1308.0796.

[11] K. Chandler, A brief proof of a maximal rank theorem for generic 2-points in projective space. Trans. Amer. Math. Soc. 353 (2000), no. 5, 1907-1920.

[12] C. Ciliberto and R. Miranda, Interpolations on curvilinear schemes, J. Algebra 203 (1998), no. 2, 677-678.

[13] R. Hartshorne and A. Hirschowitz, Droites en position générale dans $\mathbb{P}^{n}$, Algebraic Geometry, Proceedings, La Rábida 1981, 169-188, Lect. Notes in Math. 961, Springer, Berlin, 1982.

[14] A. Laface, On linear systems of curves on rational scrolls, Geom. Dedicata 90 (2002), 127-144. 